\title{
Bessel convolutions on matrix cones
}

\author{
Margit Rösler
}

\begin{abstract}
In this paper we introduce probability-preserving convolution algebras on cones of positive semidefinite matrices over one of the division algebras $\mathbb{F}=\mathbb{R}, \mathbb{C}$ or $\mathbb{H}$ which interpolate the convolution algebras of radial bounded Borel measures on a matrix space $M_{p, q}(\mathbb{F})$ with $p \geqslant q$. Radiality in this context means invariance under the action of the unitary group $U_{p}(\mathbb{F})$ from the left. We obtain a continuous series of commutative hypergroups whose characters are given by Bessel functions of matrix argument. Our results generalize wellknown structures in the rank-one case, namely the Bessel-Kingman hypergroups on the positive real line, to a higher rank setting. In a second part of the paper we study structures depending only on the matrix spectra. Under the mapping $r \mapsto \operatorname{spec}(r)$, the convolutions on the underlying matrix cone induce a continuous series of hypergroup convolutions on a Weyl chamber of type $B_{q}$. The characters are now Dunkl-type Bessel functions. These convolution algebras on the Weyl chamber naturally extend the harmonic analysis for Cartan motion groups associated with the Grassmann manifolds $U(p, q) /\left(U_{p} \times U_{q}\right)$ over $\mathbb{F}$.
\end{abstract}

\section{Introduction}

Let us start with the basic guiding example which corresponds to the rank-one case of our subsequent constructions. For a natural number $p \geqslant 2$ consider the set $M_{b}^{\text {rad }}\left(\mathbb{R}^{p}\right)$ of regular bounded Borel measures on $\mathbb{R}^{p}$ which are radial, i.e. invariant under orthogonal transformations. $M_{b}^{\mathrm{rad}}\left(\mathbb{R}^{p}\right)$ is a commutative Banach algebra with the usual convolution of measures. Transferring this structure to $\mathbb{R}_{+}=[0, \infty)$ via the mapping $x \mapsto|x|=\left(x_{1}^{2}+\cdots+x_{p}^{2}\right)^{1 / 2}$, one obtains a commutative Banach algebra of Borel measures on $\mathbb{R}_{+}$. Calculation in polar coordinates shows that its convolution $*_{p}$ is determined on point measures by

$$
\delta_{r} *_{p} \delta_{s}(f)=c_{p} \int_{0}^{\pi} f\left(\sqrt{r^{2}+s^{2}-2 r s \cos \theta}\right) \sin ^{p-2} \theta d \theta, \quad r, s \in \mathbb{R}_{+}, f \in C\left(\mathbb{R}_{+}\right)
$$

with a normalization constant $c_{p}>0$. The above assignment defines a probability measure $\delta_{r} *_{p} \delta_{s}$ not only for integer $p$ but for all real $p>1$, and it extends uniquely to a bilinear and weakly continuous convolution on the space $M_{b}\left(\mathbb{R}_{+}\right)$of regular bounded Borel measures on $\mathbb{R}_{+}$which is commutative, associative, and probability-preserving [Kin63]. The interesting point about this family of convolutions is that analytic properties which are valid for integer indices $p$, due to their origin in radial analysis on $\mathbb{R}^{p}$, remain true for general indices where no longer is any group structure present. For example, consider the normalized Bessel functions $j_{\alpha}(z)={ }_{0} F_{1}\left(\alpha+1 ;-z^{2} / 4\right)$ with index $\alpha=p / 2-1$. If $p$ is a natural number, then

$$
j_{\alpha}(|x|)=\int_{S^{p-1}} e^{-i(x \mid \xi)} d \sigma(\xi), \quad x \in \mathbb{R}^{p}
$$

Received 20 December 2005, accepted in final form 2 August 2006. 2000 Mathematics Subject Classification 33C67, 33C80, 43A62, $43 \mathrm{~A} 85$.

Keywords: Bessel functions, symmetric cones, Dunkl operators, hypergroups, product formula.

The author was supported by NWO under project number B 61-544, and by the DFG within SFB/TR-12.

This journal is (C) Foundation Compositio Mathematica 2007. 


\section{RÖSLER}

( $d \sigma$ denotes the normalized Lebesgue surface measure on the unit sphere $S^{p-1}$ ), and the homomorphism property of the exponential function entails the product formula

$$
j_{\alpha}(r) j_{\alpha}(s)=\delta_{r} *_{p} \delta_{s}\left(j_{\alpha}\right) .
$$

This formula, however, extends to arbitrary indices $p>1$, see [Wat66]. The space $\mathbb{R}_{+}$together with the convolution $*_{p}$ is a well-known example of a commutative hypergroup, called the BesselKingman hypergroup [BH95]. A hypergroup is a locally compact Hausdorff space $X$ together with a probability-preserving convolution of measures on $X$ which generalizes the measure algebra of a locally compact group; in particular, one requires a unit element and the existence of an involution on $X$ generalizing the group inverse in a suitable way; for details we refer to [Jew75] and $\S 2.3$ below. There is a rich harmonic analysis for commutative hypergroups extending the analysis on locally compact abelian groups. In particular, there is a Haar measure, a dual space, and a Fourier transform satisfying a Plancherel theorem. In our example $\left(\mathbb{R}_{+}, *_{p}\right)$ the dual space consists of the Bessel functions

$$
\left\{\varphi_{s}(r)=j_{\alpha}(r s), s \in \mathbb{R}_{+}\right\}, \quad \alpha=\frac{p}{2}-1,
$$

and the hypergroup Fourier transform is given by a Hankel transform,

$$
\widehat{f}^{p}(s)=\frac{2^{-p / 2}}{\Gamma(p / 2)} \int_{\mathbb{R}_{+}} f(r) \varphi_{s}(r) r^{p-1} d r .
$$

This extends the fact that the Fourier transform of a radial function $F(x)=f(|x|) \in L^{1}\left(\mathbb{R}^{p}\right)$ is again radial and given by a Hankel transform of $f$ with integral index $p$.

In the present paper, we generalize the Bessel convolutions described above to a higher rank setting, where the space of 'radii' is realized as a cone of positive semidefinite matrices. More precisely, we construct convolution algebras on such cones which interpolate radial convolution algebras on spaces of non-squared matrices. The setting is as follows: for $q \in \mathbb{N}$ and a natural number $p \geqslant q$ consider the space $M_{p, q}=M_{p, q}(\mathbb{F})$ of $p \times q$ matrices over one of the division algebras $\mathbb{F}=\mathbb{R}, \mathbb{C}$ or the quaternions $\mathbb{H}$. It has the structure of a Euclidean vector space with scalar product $(x \mid y)=\Re \operatorname{tr}\left(x^{*} y\right)$ (see $\S 2.1$ for the notation). A function or measure on $M_{p, q}$ is called radial if it is invariant under the action of the unitary group $U_{p}=U_{p}(\mathbb{F})$ on $M_{p, q}$ by left multiplication,

$$
U_{p} \times M_{p, q} \rightarrow M_{p, q}, \quad(u, x) \mapsto u x .
$$

Observe that this action is via orthogonal transformations, and that $x$ and $y$ are contained in the same $U_{p^{-}}$orbit if and only if $x^{*} x=y^{*} y$. Thus, the space of $U_{p^{-}}$orbits is naturally parametrized by the cone $\Pi_{q}=\Pi_{q}(\mathbb{F})$ of positive semidefinite $q \times q$-matrices over $\mathbb{F}$. Note that $\Pi_{1}(\mathbb{F})$ coincides with the non-negative real line $\mathbb{R}_{+}$. There is a radial harmonic analysis on $M_{p, q}$ which is based on polar coordinates with $\Pi_{q}$ as radial part and the Stiefel manifold

$$
\Sigma_{p, q}=\left\{x \in M_{p, q}: x^{*} x=I_{q}\right\} \cong U_{p} / U_{p-q}
$$

as a transversal manifold. In [FT87], this is developed to some extent within the general framework of symmetric cones. Indeed, the open cone

$$
\Omega_{q}=\left\{r \in \Pi_{q}: r \text { strictly positive definite }\right\}
$$

is a symmetric cone within the space $H_{q}=\left\{x \in M_{q}(\mathbb{F}): x=x^{*}\right\}$ of Hermitian $q \times q$ matrices over $\mathbb{F}$ which carries a natural Euclidean Jordan algebra structure of rank $q$; see $\S 2.1$ for details.

For each integer $q \geqslant p$ we interpret radial analysis on $M_{p, q}$ in the concise context of a commutative 'orbit hypergroup' convolution on the cone $\Pi_{q}$ which is derived from the orbit structure with respect to the action of $U_{p}$ on $M_{p, q}$. Similar to the rank-one case, the characters of this hypergroup, i.e. the multiplicative functions which make up the dual are obtained by taking the means of the 


\section{BESSEL CONVOLUTIONS ON MATRIX CONES}

exponential characters on $M_{p, q}$ over the Stiefel manifold. This implies that they are given in terms of Bessel functions $\mathcal{J}_{\mu}$ on the cone $\Pi_{q}$ with (half) integer index $\mu=p d / 2$, where $d=\operatorname{dim}_{\mathbb{R}} \mathbb{F}$. The Bessel function of index $\mu$ is a hypergeometric series of the form

$$
\mathcal{J}_{\mu}=\sum_{\lambda \geqslant 0} \frac{(-1)^{|\lambda|}}{(\mu)_{\lambda}|\lambda| !} \cdot Z_{\lambda} ;
$$

here the summation is over all partitions $\left\{\lambda=\left(\lambda_{1}, \ldots, \lambda_{q}\right) \in \mathbb{N}_{0}^{q}: \lambda_{1} \geqslant \cdots \geqslant \lambda_{q}\right\},(\mu)_{\lambda}$ is a generalized Pochhammer symbol, and the $Z_{\lambda}$ are renormalized versions of the so-called spherical polynomials associated with the underlying cone, cf. $\S 2.1$. The multiplicativity of the Bessel functions of index $\mu=p d / 2$ with respect to the associated orbit convolution on $\Pi_{q}$ expresses itself in a positive product formula. Under the technical condition $p \geqslant 2 q$, this product formula can be written in a way which allows analytic continuation with respect to the index $\mu$. We thus obtain a positive product formula for all Bessel functions with index $\mu \geqslant d(q-1 / 2)$, and an associated continuous series of hypergroup structures $*_{\mu}$ on the cone $\Pi_{q}$ whose dual is given by the functions

$$
r \mapsto \varphi_{s}(r)=\mathcal{J}_{\mu}\left(\frac{1}{4} r s^{2} r\right), \quad s \in \Pi_{q} .
$$

Actually, each hypergroup $\left(\Pi_{q}, *_{\mu}\right)$ is self-dual in a natural way; the neutral element is 0 and the involution is the identity mapping. For matrix cones, the Hankel transform of [FT87] can now be identified with the $L^{2}$-Fourier transform on the underlying hypergroup; but in addition to the results of [FT87], it is also (and primarily) defined as a Fourier transform on an $L^{1}$-convolution algebra.

Before continuing with structural aspects, let us spend some words on Bessel functions of matrix argument. They trace back to ideas of Bochner and the fundamental work of Herz [Her55] and Constantine [Con63]. Much of the interest in these functions is motivated by questions in number theory and multivariate statistics. In particular, they occur naturally in the explicit expression of non-central Wishart distributions which generalize non-central $\chi^{2}$-distributions to the higher rank case, see [Con63] and [Mui82]. Nowadays, Bessel functions of matrix argument are imbedded in rich theories of multivariable special functions. Firstly, they can be considered the ${ }_{0} F_{1}$ class among general ${ }_{p} F_{q}$-hypergeometric functions of matrix argument, where hypergeometric series are defined in terms of spherical polynomials; see [GR87] and [Jam75] for an introduction. Secondly, all of this can be done in the general setting of abstract Jordan algebras and symmetric cones, see [FK94]. In any case, the spherical polynomials depend only on the eigenvalues of their argument. Considered as functions of the spectra, they can be identified with Jack polynomials of a certain index depending on the underlying cone; this was first observed by Macdonald [Mac87]. There is a natural theory of hypergeometric expansions in terms of Jack polynomials (see [Kan93]) which encompasses the theory on symmetric cones. Finally, hypergeometric expansions of such kind are intimately related to the modern theory of hypergeometric functions associated with root systems as developed by Heckman, Opdam, Dunkl, and others. As functions of the spectra, Gaussian hypergeometric functions on a symmetric cone can be identified with hypergeometric functions associated with a root system of type $B C$ with a specific choice of parameters [BO93]. Similarly, Bessel functions on a symmetric cone can be considered as a subclass of the Bessel functions associated with reduced root systems of type $B$ in the sense of [Opd93]. The latter play a fundamental role in the theory of rational Dunkl operators [Dun89, Dun91]. This connection is taken up in the second part of the paper, where we consider structures which depend only on the spectra of the matrices from the underlying cone $\Pi_{q}$. This amounts to assuming invariance under the action of the unitary group $U_{q}=U_{q}(\mathbb{F})$ by conjugation,

$$
r \mapsto u r u^{-1}, \quad u \in U_{q} .
$$

The orbits under this action are naturally parametrized by the set $\Xi_{q}$ of possible spectra of matrices 


\section{RÖSLER}

from $\Pi_{q}$, the eigenvalues being ordered by size:

$$
\Xi_{q}=\left\{\xi=\left(\xi_{1}, \ldots, \xi_{q}\right) \in \mathbb{R}^{q}: \xi_{1} \geqslant \cdots \geqslant \xi_{q} \geqslant 0\right\} .
$$

Here $\Xi_{q}$ is a closed Weyl chamber for the reflection group $B_{q}$ which acts on $\mathbb{R}^{q}$ by permutations and sign changes of the coordinates. Via the canonical mapping from $\Pi_{q}$ onto $\Xi_{q}$ which assigns to each matrix its spectrum, the continuous series of hypergroup structures $\left(\Pi_{q}, *_{\mu}\right)$ with $\mu \geqslant d(q-1 / 2)$ induces a series of commutative hypergroup structures $\circ_{\mu}$ on the chamber $\Xi_{q}$. The transfer is established by means of so-called orbital mappings. The convolution, Haar measure, and dual space of each hypergroup on the chamber are made explicit. In particular, the Haar measure of $\left(\Xi_{q}, \circ_{\mu}\right)$ is (up to a constant factor) given by $h_{\mu}(\xi) d \xi$ where

$$
h_{\mu}(\xi)=\prod_{i=1}^{q} \xi_{i}^{2 \gamma+1} \prod_{i<j}\left(\xi_{i}^{2}-\xi_{j}^{2}\right)^{d}, \quad \gamma=\mu-\frac{d}{2}(q-1)-1 .
$$

The hypergroup characters turn out to be certain Dunkl-type Bessel functions

$$
\xi \mapsto J_{k}^{B}(\xi, i \eta), \quad \eta \in \Xi_{q}
$$

associated with the $B_{q}$ root system $\left\{ \pm e_{i}, \pm e_{i} \pm e_{j}\right\} \subset \mathbb{R}^{q}$. Here $k$ is a parameter on the root system which is constant on each subset of roots corresponding to a conjugacy class of reflections; in our situation it is given by $k=\left(k_{1}, k_{2}\right)$ with

$$
k_{1}=\mu-\frac{d}{2}(q-1)-\frac{1}{2} \quad \text { on } \pm e_{i} ; \quad k_{2}=\frac{d}{2} \quad \text { on } \pm e_{i} \pm e_{j} .
$$

The hypergroup convolution on the Weyl chamber matches the generalized Dunkl translation [Rös98] for Weyl group invariant functions, and we have an interpretation of the Dunkl transform as a hypergroup Fourier transform. It is an important observation in this context that Bessel functions on an arbitrary symmetric cone of rank $q$ are given by Dunkl Bessel functions of type $B_{q}$, see Corollary 4.6 .

In the geometric cases $\mu=p d / 2$, the support of the probability measure $\delta_{\xi} \circ_{\mu} \delta_{\eta}$ on $\Xi_{q}$ describes the set of possible singular spectra of sums $x+y$ with matrices $x, y \in M_{p, q}$ having given singular spectra $\xi$ and $\eta$. Further, the characters are just the bounded spherical functions of the Euclidean-type Riemannian symmetric space $\left(U_{p} \times U_{q}\right) \ltimes M_{p, q} /\left(U_{p} \times U_{q}\right)$ associated with the Grassmann manifold $U(p, q) / U_{p} \times U_{q}$. For general $\mu$ they are characterized, within the theory of rational Dunkl operators, as the unique analytic solution of a so-called Bessel system; see [Opd93]. It is conjectured that for arbitrary root systems and non-negative multiplicities, the associated Dunkl-type Bessel functions satisfy a positive product formula and can be characterized as the characters of a commutative hypergroup structure on the underlying Weyl chamber. The three continuous series $(d=1,2,4)$ for $B_{q}$ obtained in this paper are, to the best of our knowledge, the first affirmative examples beyond the group cases associated with Cartan motion groups of reductive symmetric spaces. Some background, together with further partial results, is given in [Rös03a].

The organization of this paper is as follows. In $\S 2$, background on symmetric cones and Bessel functions on cones, as well as some hypergroup analysis are provided. Section 3 is devoted to the study of Bessel convolutions on matrix cones. In $\S 3.1$, orbit convolutions derived from matrix spaces $M_{p, q}$ are considered. In $\S 3.3$, the corresponding product formula for the involved Bessel functions is analytically extended with respect to the index, and in $\S 3.4$ the associated series of hypergroup convolutions on the cone are studied. In particular, their Haar measure and the dual are determined. In $\S 3.5$ we analyse an interesting critical index. Section 4 is devoted to the induced convolution algebras on a Weyl chamber of type $B$. They are derived in $\S 4.1$ from the convolutions on the matrix cones and are then, in the two final subsections, put into relation to rational Dunkl theory. 


\section{BESSEL CONVOLUTIONS ON MATRIX CONES}

\section{Preliminaries}

In this introductory section we provide some relevant background on symmetric cones, in particular matrix cones, and about Bessel functions on such cones. In the main part of the paper we shall introduce orbit convolutions and their 'interpolations' on matrix cones within the framework of hypergroup theory. A short account on the relevant notions and facts is therefore included in the present section. For a general background on hypergroups, the reader is referred to the fundamental article [Jew75] (where the notion 'convo' is being used instead of 'hypergroup'), or to the monograph [BH95]. An excellent reference for analysis on symmetric cones is [FK94]; for special functions on matrix cones see also [GR87] as well as the classical papers of Herz [Her55], James [Jam75], and Constantine [Con63].

\subsection{Analysis on symmetric cones}

Let $\mathbb{F}$ be one of the division algebras $\mathbb{F}=\mathbb{R}, \mathbb{C}$ or the quaternions $\mathbb{H}$. We denote by $t \mapsto \bar{t}$ the usual conjugation in $\mathbb{F}$ and by $\mathfrak{R} t=\frac{1}{2}(t+\bar{t})$ the real part of $t \in \mathbb{F}$. Consider the set of Hermitian $q \times q$-matrices over $\mathbb{F}$,

$$
H_{q}=H_{q}(\mathbb{F})=\left\{x \in M_{q}(\mathbb{F}): x=x^{*}\right\} ; \quad x^{*}=\bar{x}^{t} .
$$

We regard $H_{q}$ as a Euclidean vector space with scalar product $(x \mid y)=\mathfrak{R t r}(x y)$, where tr denotes the trace on $M_{q}(\mathbb{F})$. The dimension of $H_{q}$ over $\mathbb{R}$ is

$$
n=q+\frac{d}{2} q(q-1), \quad d=\operatorname{dim}_{\mathbb{R}} \mathbb{F} .
$$

With the above scalar product and the Jordan product $x \circ y=\frac{1}{2}(x y+y x)$, the matrix space $H_{q}$ becomes a Euclidean Jordan algebra with unit $I=I_{q}$, the unit matrix. The rank of $H_{q}$, that is the number of elements of each Jordan frame in $H_{q}$, is $q$.

The set $\Omega_{q}=\Omega_{q}(\mathbb{F})$ of those matrices from $H_{q}$ which are positive definite is a symmetric cone. Recall that a symmetric cone $\Omega$ is a proper, non-empty convex cone in a finite-dimensional Euclidean vector space $V$ which is self-dual and homogeneous in the sense that its group of linear automorphisms acts transitively. Let $G$ denote the connected component of this group. Then $K=$ $G \cap O(V)$ is a maximal compact subgroup of $G$ and $\Omega \cong G / K$, a Riemannian symmetric space. The matrix cones are realized as $\Omega_{q}(\mathbb{F}) \cong \mathrm{GL}_{q}(\mathbb{F}) / U_{q}(\mathbb{F})$. Hereby $\mathrm{GL}_{q}(\mathbb{F})$ acts via $r \mapsto g r g^{*}$, which reduces to conjugation when restricted to the unitary subgroup $U_{q}(\mathbb{F})$. Our main interest will be in the closure of $\Omega_{q}$ relative to $H_{q}$ which coincides with the set of positive semidefinite matrices over $\mathbb{F}$,

$$
\Pi_{q}=\Pi_{q}(\mathbb{F})=\left\{x^{*} x: x \in H_{q}\right\}=\left\{x^{2}: x \in H_{q}\right\} .
$$

For a general Euclidean (i.e. formally real) Jordan algebra $V$, the interior $\Omega$ of the set $\left\{x^{2}: x \in V\right\}$ is a symmetric cone, and each symmetric cone in a finite-dimensional Euclidean vector space $V$ can be realized in such a way. For details see [FK94, §III.3]. The simple Euclidean Jordan algebras correspond to the irreducible symmetric cones and are classified. Up to isomorphism, there are the above series $H_{q}(\mathbb{F})$ with $\mathbb{F}=\mathbb{R}, \mathbb{C}, \mathbb{H}$, the exceptional Jordan algebra $H_{3}(\mathbb{O})$, as well as one infinite series of rank-two algebras corresponding to the Lorentz cones

$$
\Lambda_{n}=\left\{\left(x^{\prime}, x_{n}\right) \in \mathbb{R} \times \mathbb{R}^{n-1}: x_{n}^{2}-\left|x^{\prime}\right|^{2}>0, x_{n}>0\right\} .
$$

Let $V$ be a simple Euclidean Jordan algebra of rank $q$ and real dimension $n$, let $e$ be the unit of $V$ and $\Omega$ the associated symmetric cone. We recall that for each $x \in V$ there is a Jordan frame $\left\{e_{1}, \ldots, e_{q}\right\}$ such that $x=\sum_{i=1}^{q} \xi_{i} e_{i}$ with real numbers $\xi_{i}$. Up to ordering, the $\xi_{i}$ are uniquely determined and are called the eigenvalues of $x$. Note that $x \in \Omega$ if and only if all its eigenvalues 


\section{RÖSLER}

are positive. The trace and determinant of $x$ are defined by

$$
\operatorname{tr} x=\sum_{i=1}^{q} \xi_{i}, \quad \Delta(x)=\prod_{i=1}^{q} \xi_{i} .
$$

In the Jordan algebras $H_{q}(\mathbb{F})$, the function $\Delta$ coincides with the usual determinant $\operatorname{det}_{\mathbb{F}}$ if $\mathbb{F}=\mathbb{R}$ or $\mathbb{C}$, while for $\mathbb{F}=\mathbb{H}$ it coincides with the Dieudonné determinant, i.e. $\Delta(x)=\left(\operatorname{det}_{\mathbb{C}} x\right)^{1 / 2}$ when $x$ is considered as a complex matrix in the usual way.

Recall that a $q$-tuple $\lambda=\left(\lambda_{1}, \ldots, \lambda_{q}\right) \in \mathbb{N}_{0}^{q}$ is called a partition if $\lambda_{1} \geqslant \cdots \geqslant \lambda_{q} \geqslant 0$. Following a standard convention, we shall often write $\lambda \geqslant 0$ to indicate that $\lambda$ is a partition. For $\mu \in \mathbb{C}$, a parameter $\alpha>0$ and a partition $\lambda=\left(\lambda_{1}, \ldots, \lambda_{q}\right) \in \mathbb{N}_{0}^{q}$, the generalized Pochhammer symbol $(\mu)_{\lambda}^{\alpha}$ is defined by

$$
(\mu)_{\lambda}^{\alpha}=\prod_{j=1}^{q}\left(\mu-\frac{1}{\alpha}(j-1)\right)_{\lambda_{j}} .
$$

The basic functions for the harmonic analysis on a symmetric cone $\Omega$ are the so-called spherical polynomials. These are just the polynomial spherical functions of $\Omega$ considered as a symmetric space $G / K$. They are indexed by partitions $\lambda \in \mathbb{N}_{0}^{q}$ and defined by

$$
\Phi_{\lambda}(x)=\int_{K} \Delta_{\lambda}(k x) d k, \quad x \in V
$$

where $d k$ is the normalized Haar measure on $K$ and $\Delta_{\lambda}$ is the generalized power function on $V$,

$$
\Delta_{\lambda}(x)=\Delta_{1}(x)^{\lambda_{1}-\lambda_{2}} \Delta_{2}(x)^{\lambda_{2}-\lambda_{3}} \cdots \Delta_{q}(x)^{\lambda_{q}} .
$$

The $\Delta_{i}(x)$ are the principal minors of $\Delta(x)$, see [FK94] for details. The power function $\Delta_{\lambda}$ is a homogeneous polynomial of degree $|\lambda|=\lambda_{1}+\cdots+\lambda_{q}$ which is positive on $\Omega$. Note that $\Phi_{\lambda}(e)=1$. Further, $\Phi_{\lambda}$ is $K$-invariant and therefore depends only on the eigenvalues of its argument. For the matrix cones over $\mathbb{R}$ the $\Phi_{\lambda}$ are known as zonal polynomials, for those over $\mathbb{C}$ they coincide with Schur polynomials. Actually, for each symmetric cone the associated spherical polynomials are given in terms of Jack polynomials [Sta89]. This was first observed by Macdonald [Mac87] (see also [Far92] and the notes in [FK94, ch. XI]). More precisely, let $C_{\lambda}^{\alpha}$ denote the Jack polynomials in $q$ variables and with parameter $\alpha>0$, indexed by partitions $\lambda \in \mathbb{N}_{0}^{q}$. We assume them to be normalized such that

$$
\left(\xi_{1}+\cdots+\xi_{q}\right)^{k}=\sum_{\lambda \geqslant 0,|\lambda|=k} C_{\lambda}^{\alpha}(\xi) \quad \forall k \in \mathbb{N}_{0}
$$

(cf. [Kan93]). For an irreducible symmetric cone $\Omega \subseteq V$ as above, let $d$ be the Peirce constant given by $d=\operatorname{dim}_{\mathbb{R}} \mathbb{F}$ if $\Omega$ is a matrix cone over $\mathbb{F}$ while $d=n-2$ if $\Omega$ is the Lorentz cone $\Lambda_{n}$. Consider the renormalized spherical polynomials

$$
Z_{\lambda}(x):=d_{\lambda} \frac{|\lambda| !}{(n / q)_{\lambda}^{\alpha}} \cdot \Phi_{\lambda}(x)
$$

where $\alpha=2 / d$ and $d_{\lambda}$ denotes the dimension of the vector space of polynomials on $V^{\mathbb{C}}$ (the complexification of $V$ ), which is generated by the elements $z \mapsto \Delta_{\lambda}\left(g^{-1} z\right), g \in G$; cf. [FK94, §XI.5]. Then for $x \in V$ with eigenvalues $\xi=\left(\xi_{1}, \ldots, \xi_{q}\right)$ we have

$$
Z_{\lambda}(x)=C_{\lambda}^{\alpha}(\xi), \quad \alpha=\frac{2}{d} .
$$

In normalization constants, we also need the gamma function of $\Omega$,

$$
\Gamma_{\Omega}(z)=\int_{\Omega} e^{-\operatorname{tr} x} \Delta(x)^{z-n / q} d x
$$




\section{BESSEL CONVOLUTIONS ON MATRIX CONES}

which converges absolutely for $z \in \mathbb{C}$ with $\mathfrak{R} z>n / q-1$ and can be written in terms of the classical gamma function as

$$
\Gamma_{\Omega}(z)=(2 \pi)^{(n-q) / 2} \prod_{j=1}^{q} \Gamma\left(z-\frac{d}{2}(j-1)\right)
$$

see [FK94, ch. VII.1].

\subsection{Bessel functions on a symmetric cone}

Hypergeometric expansions in terms of spherical polynomials have a long history in multivariate statistics, tracing back to the work of Herz [Her55], James [Jam75], and Constantine [Con63]. They are important in the study of Wishart distributions and for questions related to total positivity [GR89]. In view of the above connection between spherical polynomials and Jack polynomials it is natural to treat these classes of functions in the more general framework of multivariable hypergeometric functions based on Jack polynomial expansions (see [Kan93]). In our context, only hypergeometric functions of type ${ }_{0} F_{1}$ (which are Bessel functions) will be relevant.

For an arbitrary parameter $\alpha>0$ and an index $\mu \in \mathbb{C}$ satisfying $(\mu)_{\lambda}^{\alpha} \neq 0$ for all partitions $\lambda \in \mathbb{N}_{0}^{q}$, the generalized hypergeometric function ${ }_{0} F_{1}^{\alpha}(\mu ; \cdot)$ on $\mathbb{C}^{q}$ is defined by

$$
{ }_{0} F_{1}^{\alpha}(\mu ; \xi)=\sum_{\lambda \geqslant 0} \frac{1}{(\mu)_{\lambda}^{\alpha}|\lambda| !} \cdot C_{\lambda}^{\alpha}(\xi)
$$

It is known [Kan93] that this series converges absolutely for all $\xi \in \mathbb{C}^{q}$. Similarly, a ${ }_{0} F_{1}$-hypergeometric function of two arguments is defined by

$$
{ }_{0} F_{1}^{\alpha}(\mu ; \xi, \eta)=\sum_{\lambda \geqslant 0} \frac{1}{(\mu)_{\lambda}^{\alpha}|\lambda| !} \cdot \frac{C_{\lambda}^{\alpha}(\xi) C_{\lambda}^{\alpha}(\eta)}{C_{\lambda}^{\alpha}(\mathbf{1})}, \quad \mathbf{1}=(1, \ldots, 1) .
$$

Now suppose that $\alpha=2 / d$ where $d$ is the Peirce constant of a simple Euclidean Jordan algebra $V$ of rank $q$ corresponding to the symmetric cone $\Omega$. Then the Bessel function ${ }_{0} F_{1}^{\alpha}(\mu ; \cdot)$ essentially coincides with the Bessel function $\mathcal{J}_{\mu}$ associated with $\Omega$ in the sense of [FK94]. Indeed, the latter is given by

$$
\mathcal{J}_{\mu}(x)=\sum_{\lambda \geqslant 0} \frac{(-1)^{|\lambda|}}{(\mu)_{\lambda}^{\alpha}|\lambda| !} Z_{\lambda}(x) \quad \text { for } x \in V ; \quad \alpha=\frac{2}{d} .
$$

Thus, for $x \in V$ with eigenvalues $\xi=\left(\xi_{1}, \ldots, \xi_{q}\right)$,

$$
\mathcal{J}_{\mu}(x)={ }_{0} F_{1}^{2 / d}(\mu ;-\xi) .
$$

If $q=1$, then $\mathcal{J}_{\mu}$ is independent of $d$ and we have

$$
\mathcal{J}_{\mu}\left(\frac{x^{2}}{4}\right)=j_{\mu-1}(x)
$$

with the one-variable Bessel function $j_{\mu-1}$ as in the introduction. In $\S 4.3$ we also work with Bessel functions of two arguments $x, y \in V$,

$$
\mathcal{J}_{\mu}(x, y):=\sum_{\lambda \geqslant 0} \frac{(-1)^{|\lambda|}}{(\mu)_{\lambda}^{\alpha}|\lambda| !} \frac{Z_{\lambda}(x) Z_{\lambda}(y)}{Z_{\lambda}(e)} .
$$

For $x, y \in V$ with eigenvalues $\xi=\left(\xi_{1}, \ldots, \xi_{q}\right)$ and $\eta=\left(\eta_{1}, \ldots, \eta_{q}\right)$ respectively, we have

$$
\mathcal{J}_{\mu}(x, y)={ }_{0} F_{1}^{2 / d}(\mu ; i \xi, i \eta) .
$$

We shall see in the following that these functions are Bessel functions of Dunkl type. The following estimate is especially useful for large indices $\mu$. 


\section{RÖSLER}

Lemma 2.1. For $\alpha>0$ let ${ }_{0} F_{1}^{\alpha}(\mu ; \cdot)$ denote the associated generalized Bessel function of index $\mu$ on $\mathbb{C}^{q}$. Suppose that $\mathfrak{R} \mu>\frac{1}{\alpha}(q-1)$. Then for $\xi=\left(\xi_{1}, \ldots, \xi_{q}\right) \in \mathbb{C}^{q}$,

$$
\left|{ }_{0} F_{1}^{\alpha}(\mu ; \xi)\right| \leqslant e^{\left|\xi_{1}\right|+\cdots+\left|\xi_{q}\right|} .
$$

Proof. For $n \in \mathbb{N}_{0}$ and $z \in \mathbb{C}$ with $\mathfrak{R} z>0$ we have

$$
\left|\frac{\Gamma(z)}{\Gamma(z+n)}\right| \leqslant \frac{\Gamma(\Re z)}{\Gamma(\Re z+n)} \leqslant 1 .
$$

Therefore, $\left|(\mu)_{\lambda}^{\alpha}\right| \geqslant\left|(\Re \mu)_{\lambda}^{\alpha}\right| \geqslant 1$. Moreover, it is known that the coefficients of the Jack polynomial $C_{\lambda}^{\alpha}$ in its monomial expansion are all non-negative (see [KS97]). This implies that

$$
\left|C_{\lambda}^{\alpha}(\xi)\right| \leqslant C_{\lambda}^{\alpha}\left(\left|\xi_{1}\right|, \ldots,\left|\xi_{q}\right|\right)
$$

Using relation (2.1), we therefore obtain

$$
\left|{ }_{0} F_{1}^{\alpha}(\mu ; \xi)\right| \leqslant \sum_{\lambda \geqslant 0} \frac{1}{|\lambda| !} C_{\lambda}^{\alpha}\left(\left|\xi_{1}\right|, \ldots,\left|\xi_{q}\right|\right)=e^{\left|\xi_{1}\right|+\cdots+\left|\xi_{q}\right|} .
$$

Corollary 2.2. Let $\mathcal{J}_{\mu}$ denote the Bessel function of index $\mu$ associated with the symmetric cone $\Omega$ inside the Jordan algebra $V$ of rank $q$. Suppose that $\mathfrak{R} \mu>\frac{d}{2}(q-1)$. Then for $x \in V$ with eigenvalues $\xi_{1}, \ldots, \xi_{q}$,

$$
\left|\mathcal{J}_{\mu}(x)\right| \leqslant e^{\left|\xi_{1}\right|+\cdots+\left|\xi_{q}\right|}
$$

We mention at this point that for $\Re \mu>d(q-1)+1$ and $a \in \Omega$, the Bessel function on $\Omega$ has the absolutely convergent integral representation (an inverse Laplace integral)

$$
\mathcal{J}_{\mu}(r)=\frac{\Gamma_{\Omega}(\mu)}{(2 \pi i)^{n}} \int_{a+i V} e^{\operatorname{tr} w} e^{-\left(w^{-1} \mid r\right)} \Delta(w)^{-\mu} d w \quad(r \in \Omega)
$$

see [FK94, Proposition XV.2.2]. This integral was originally used by Herz [Her55] to define Bessel functions on the cones of positive definite matrices over $\mathbb{R}$; see also [FT87] and [Dib90] for arbitrary symmetric cones. Corollary 2.2 slightly improves the order estimate for $\mathcal{J}_{\mu}$ given in [Her55, p. 486] for $\mathbb{F}=\mathbb{R}$.

\subsection{Hypergroups}

We start with some notation: for a locally compact Hausdorff space $X$, let $M_{b}(X)$ denote the Banach space of all bounded regular (complex) Borel measures on $X$ with total variation norm, and $M^{1}(X) \subset M_{b}(X)$ the set of all probability measures. With $\delta_{x}$ we denote the point measure in $x \in X$. We use the notation $C(X), C_{b}(X)$, and $C_{c}(X)$ for the spaces of continuous complex-valued functions on $X$, those which are bounded, and those having compact support, respectively. Further, $C_{0}(X)$ is the set of functions from $C(X)$ which vanish at infinity.

Definition 2.3. A hypergroup $(X, *)$ is a locally compact Hausdorff space $X$ with a bilinear and associative convolution $*$ on $M_{b}(X)$ with the following properties.

(1) The map $(\mu, \nu) \mapsto \mu * \nu$ is weakly continuous, i.e. with respect to the topology induced by $C_{b}(X)$.

(2) For all $x, y \in X$, the product $\delta_{x} * \delta_{y}$ of point measures is a compactly supported probability measure on $X$.

(3) The mapping $(x, y) \mapsto \operatorname{supp}\left(\delta_{x} * \delta_{y}\right)$ from $X \times X$ into the space of non-empty compact subsets of $X$ is continuous with respect to the Michael topology (see [Jew75]).

(4) There is a neutral element $e \in X$, satisfying $\delta_{e} * \delta_{x}=\delta_{x} * \delta_{e}=\delta_{x}$ for all $x \in X$. 


\section{BESSEL CONVOLUTIONS ON MATRIX CONES}

(5) There is a continuous involutive automorphism $x \mapsto \bar{x}$ on $X$ such that $\delta_{\bar{x}} * \delta_{\bar{y}}=\left(\delta_{y} * \delta_{x}\right)^{-}$and $x=\bar{y} \Longleftrightarrow e \in \operatorname{supp}\left(\delta_{x} * \delta_{y}\right)$. (For $\mu \in M_{b}(X)$, the measure $\mu^{-}$is given by $\mu^{-}(A)=\mu(\bar{A})$ for Borel sets $A \subseteq X$.)

A hypergroup $(X, *)$ is called commutative if its convolution is commutative.

For a hypergroup $(X, *)$, the space $\left(M_{b}(X), *\right)$ is a Banach algebra with unit $\delta_{e}$. Note that by the density of the finitely supported measures in $M_{b}(X)$, the convolution is uniquely determined as soon as it is given for point measures.

Of course, every locally compact group is a hypergroup with the usual group convolution. In our context, only commutative hypergroups will be relevant. Of particular interest is the following.

Example 2.4 (Jewett [Jew75, ch. 8]). Let $(G,+)$ be a locally compact abelian group and $K$ a compact group acting continuously on $G$ by automorphisms of $G$. Then the space $G^{K}=\{K . x: x \in G\}$ of $K$-orbits in $G$ is a locally compact Hausdorff space with the quotient topology and becomes a commutative hypergroup with the (natural) definition

$$
\left(\delta_{K . x} * \delta_{K . y}\right)(f)=\int_{K} f(K .(x+k y)) d k, \quad f \in C\left(G^{K}\right) .
$$

The hypergroup $\left(G^{K}, *\right)$ is called an orbit hypergroup; its neutral element is $K .0=0$ and the involution is $(K . x)^{-}=K \cdot(-x)$.

In the following, we collect some further ingredients underlying the harmonic analysis on a commutative hypergroup $X$. There exists (up to normalization) a unique Haar measure $\omega$ on $X$, i.e. a positive Radon measure satisfying

$$
\int_{X} f(x * y) d \omega(y)=\int_{X} f(y) d \omega(y) \quad \forall x \in X, f \in C_{c}(X) .
$$

Here and in the following we use the convenient notation

$$
f(x * y):=\int_{X} f d\left(\delta_{x} * \delta_{y}\right)=\left(\delta_{x} * \delta_{y}\right)(f) .
$$

Similar to the dual of a locally compact abelian group, one defines the dual space of $X$ as

$$
\widehat{X}:=\left\{\varphi \in C_{b}(X): \varphi \neq 0, \varphi(\bar{x})=\overline{\varphi(x)} \text { and } \varphi(x * y)=\varphi(x) \varphi(y) \forall x, y \in X\right\} .
$$

This is a locally compact Hausdorff space with the topology of compact-uniform convergence. The elements of $\widehat{X}$ are also called the characters of $X$. They are the constituting functions for harmonic analysis on $(X, *)$. The Fourier transform on $M_{b}(X)$ is defined by

$$
\widehat{\mu}(\varphi):=\int_{X} \overline{\varphi(x)} d \mu(x), \quad \varphi \in \widehat{X}
$$

and on $L^{1}(X, \omega)$ by $\widehat{f}:=\widehat{f \omega}$. The Fourier transform is injective, and there exists a unique positive Radon measure $\pi$ on $\widehat{X}$, called the Plancherel measure of $(X, *)$, such that $f \mapsto \widehat{f}$ extends to an isometric isomorphism from $L^{2}(X, \omega)$ onto $L^{2}(\widehat{X}, \pi)$. While the Haar measure $\omega$ has full support, the support of $\pi$ may be a proper subset of $\widehat{X}$ only.

Example 2.5 (Continuation of Example 2.4). For a Haar measure $m$ on the group $G$, the image measure of $m$ under the canonical map $\pi: G \rightarrow G^{K}$ provides a Haar measure on the orbit hypergroup $G^{K}$. Further, it is easily seen that the functions

$$
\varphi_{\alpha}(K . x):=\int_{K} \alpha(k . x) d k, \quad \alpha \in \widehat{G},
$$

belong to the dual of $G^{K}$. Actually, we have the following. 


\section{RÖSLER}

LEMma 2.6. We have

(1) $\widehat{G^{K}}=\left\{\varphi_{\alpha}: \alpha \in \widehat{G}\right\}$;

(2) $\varphi_{\alpha}=\varphi_{\alpha^{\prime}}$ if and only if $\alpha$ and $\alpha^{\prime}$ are contained in the same orbit under the dual action of $K$ on $\widehat{G}$, given by $(k \cdot \alpha)(x)=\alpha\left(k^{-1} \cdot x\right)$.

Proof. It is known that $\widehat{G^{K}}$ can be identified with the extremal points of the set

$$
\Xi=\{\beta \in C(G): \beta \text { positive definite and } K \text {-invariant with } \beta(0)=1\} ;
$$

the identification is given by $\beta \mapsto \varphi_{\beta}, \varphi_{\beta}(K . x)=\beta(x)$ for $x \in G$, see [Ros78]. By Bochner's theorem, each $\beta \in \Xi$ is of the form $\beta(x)=\int_{\widehat{G}} \alpha(x) d \mu(\alpha)$ with some $K$-invariant probability measure $\mu$ on $\widehat{G}$. In this way, the extremal points of $\Xi$ correspond to the measures of the form $\mu_{\alpha}=\int_{K} \delta_{k . \alpha} d k$ with $\alpha \in \widehat{G}$. Further, $\mu_{\alpha}=\mu_{\alpha^{\prime}}$ if and only if $\alpha$ and $\alpha^{\prime}$ belong to the same $K$-orbit in $\widehat{G}$.

A commutative hypergroup $(X, *)$ is called self-dual if there exists a homeomorphism $\Psi: X \rightarrow \widehat{X}$ such that

$$
\Psi(x)(z) \Psi(y)(z)=\int_{X} \Psi(w)(z) d\left(\delta_{x} * \delta_{y}\right)(w) \quad \forall x, y, z \in X .
$$

In that case, $\widehat{X}$ is a dual hypergroup in the sense of [Jew75, § 12.4] where the convolution product $\delta_{\Psi(x)} * \delta_{\Psi(y)}$ is just the image measure of $\delta_{x} * \delta_{y}$ under $\Psi$. It is easily checked that the image measure $\Psi(\omega)$ of the Haar measure $\omega$ on $X$ is a Haar measure on $\widehat{X}$. Thus, by [Jew75, Theorem 12.4A], $\Psi(\omega)$ coincides up to a multiplicative constant with the Plancherel measure $\pi$ of $X$.

\section{Convolution structures associated with Bessel functions on a matrix cone}

\subsection{Orbit hypergroups on a matrix cone}

For natural numbers $p \geqslant q$, consider the matrix space $M_{p, q}=M_{p, q}(\mathbb{F})$ of $p \times q$-matrices over $\mathbb{F}$. We regard $M_{p, q}$ as a real vector space, equipped with the Euclidean scalar product $(x \mid y):=\mathfrak{R} \operatorname{tr}\left(x^{*} y\right)$ and Hilbert-Schmidt norm $\|x\|=\sqrt{\operatorname{tr}\left(x^{*} x\right)}$. The unitary group $U_{p}=U_{p}(\mathbb{F})$ acts on $M_{p, q}$ by left multiplication,

$$
U_{p} \times M_{p, q} \rightarrow M_{p, q}, \quad(u, x) \mapsto u x .
$$

The orbit space $M_{p, q}^{U_{p}}$ for this action can be identified with the space $\Pi_{q}=\Pi_{q}(\mathbb{F})$ of positive semidefinite $q \times q$ matrices over $\mathbb{F}$ via

$$
U_{p} . x \mapsto \sqrt{x^{*} x}=:|x| .
$$

Here for $r \in \Pi_{q}, \sqrt{r}$ denotes the unique positive semidefinite square root of $r$. It is easy to see that the above bijection becomes a homeomorphism when $M_{p, q}^{U_{p}}$ is equipped with the quotient topology and $\Pi_{q}$ with the subspace topology induced from $M_{q}$. (Indeed, both the canonical map $x \mapsto U_{p} . x$ from $M_{p, q}$ onto $M_{p, q}^{U_{p}}$ and the mapping $x \mapsto \sqrt{x^{*} x}, M_{p, q} \rightarrow \Pi_{q}$ are open and continuous.) Note that the Stiefel manifold

$$
\Sigma_{p, q}=\left\{x \in M_{p, q}: x^{*} x=I_{q}\right\}
$$

is the orbit of the block matrix

$$
\sigma_{0}:=\left(\begin{array}{c}
I_{q} \\
0
\end{array}\right) \in M_{p, q}
$$

Before calculating the orbit hypergroup convolution for the above action, let us recall the basic aspects of radial analysis in $M_{p, q}$ as developed in [FT87]. As in the introduction, radiality here means invariance under the above action of $U_{p}$; a function $F$ on $M_{p, q}$ is radial if and only if it is of the form $F(x)=f(|x|)$ for some $f: \Pi_{q} \rightarrow \mathbb{C}$. Suitable polar coordinates in $M_{p, q}$ are defined as 


\section{BESSEL CONVOLUTIONS ON MATRIX CONES}

follows: let $d \sigma$ denote the $U_{p}$-invariant measure on $\Sigma_{p, q}$, normalized according to $\int_{\Sigma_{p, q}} d \sigma=1$, and let

$$
M_{p, q}^{\prime}=\left\{x \in M_{p, q}: \Delta\left(x^{*} x\right) \neq 0\right\}
$$

which is open and dense in $M_{p, q}$. Then the mapping

$$
\Omega_{q} \times \Sigma_{p, q} \rightarrow M_{p, q}^{\prime}, \quad(r, \sigma) \mapsto \sigma \sqrt{r}
$$

is a diffeomorphism, and for integrable functions $f: M_{p, q} \rightarrow \mathbb{C}$ one has

$$
\int_{M_{p, q}} f(x) d x=C_{p, q} \int_{\Omega_{q}} \int_{\Sigma_{p, q}} f(\sigma \sqrt{r}) \Delta(r)^{\gamma} d r d \sigma
$$

with

$$
C_{p, q}=\frac{\pi^{d p q / 2}}{\Gamma_{\Omega_{q}}(d p / 2)} \quad \text { and } \quad \gamma=\frac{d p}{2}-\frac{n}{q} .
$$

As before, $n$ is the real dimension of $H_{q}$. Our notion slightly differs from that of [FT87] (and the monograph [FK94]); it is adapted to the left action (1.1) of $U_{p}$ on $M_{p, q}$ while the notion of [FT87] would require to have $U_{p}$ acting from the right on $M_{q, p}$. As Lebesgue measure on $M_{p, q}$ is $U_{p}$-invariant, the Fourier transform of a radial function in $L^{1}\left(M_{p, q}\right)$ is again radial, and also the convolution of two radial functions is radial; both can be calculated by the use of polar coordinates, cf. [FT87]. We shall come back to this shortly. Following Example 2.4, we obtain the orbit hypergroup convolution

$$
\left(\delta_{r} * \delta_{s}\right)(f)=\int_{U_{p}} f\left(\left|\sigma_{0} r+u \sigma_{0} s\right|\right) d u, \quad r, s \in \Pi_{q} .
$$

The image measure of $d u$ under the mapping $U_{p} \rightarrow \Sigma_{p, q}, u \mapsto u \sigma_{0}$ is $U_{p}$-invariant and therefore coincides with the $U_{p}$-invariant measure $d \sigma$. Hence,

$$
\left(\delta_{r} * \delta_{s}\right)(f)=\int_{\Sigma_{p, q}} f\left(\left|\sigma_{0} r+\sigma s\right|\right) d \sigma=\int_{\Sigma_{p, q}} f\left(\sqrt{r^{2}+s^{2}+r \widetilde{\sigma} s+(r \widetilde{\sigma} s)^{*}}\right) d \sigma
$$

where $\tilde{\sigma}=\sigma_{0}^{*} \sigma$ is the $q \times q$-matrix whose rows are given by the first $q$ rows of $\sigma$. Actually, this convolution depends on $p$ (and $q$ ), which we surpress for the moment. The neutral element of the orbit hypergroup $\left(\Pi_{q}, *\right)$ is 0 , and the involution is the identity mapping (because $x \in M_{p, q}$ and $-x$ are in the same $U_{p}$-orbit). Further, according to Lemma 2.6 , the dual space of $\left(\Pi_{q}, *\right)$ consists of the functions $\varphi_{s}, s \in \Pi_{q}$ with

$$
\varphi_{s}(r)=\int_{U_{p}} e^{-i\left(u \sigma_{0} r \mid \sigma_{0} s\right)} d u=\int_{\Sigma_{p, q}} e^{-i\left(\sigma \mid \sigma_{0} s r\right)} d \sigma .
$$

These are Bessel functions. Indeed, according to [FK94, Proposition XVI.2.3] we have, for $x \in M_{p, q}$, the identity

$$
\int_{\Sigma_{p, q}} e^{-i(\sigma \mid x)} d \sigma=\mathcal{J}_{\mu}\left(\frac{1}{4} x^{*} x\right), \quad \mu=\frac{p d}{2}
$$

where $\mathcal{J}_{\mu}$ is the Bessel function of index $\mu$ associated with the symmetric cone $\Omega_{q}$ as in $\S 2.2$. Thus,

$$
\varphi_{s}(r)=\mathcal{J}_{\mu}\left(\frac{1}{4} r s^{2} r\right) .
$$

We mention at this point that up to a constant factor, $\varphi_{s}(r)$ coincides with the Bessel function $J\left(r^{2}, s^{2}\right)$ in [FT87]. As $\mathcal{J}_{\mu}(x)$ depends only on the eigenvalues of $x$ and as the matrices $r s^{2} r$ and $s r^{2} s$ have the same eigenvalues, we see that

$$
\varphi_{s}(r)=\varphi_{r}(s) \quad \forall r, s \in \Pi_{q} .
$$

This implies that the hypergroup $\left(\Pi_{q}, *\right)$ is self-dual via $r \mapsto \varphi_{r}$. 


\section{RÖSLER}

DEFinition 3.1. In order to stress that the convolution $*$ of the orbit hypergroup $\Pi_{q} \cong M_{p, q}^{U_{p}}$ depends on $p$ or, equivalently, on the index $\mu=p d / 2$, we denote it by $*_{\mu}$ and write $\Pi_{q, \mu}$ for the orbit hypergroup $\left(\Pi_{q}, *_{\mu}\right)$.

A Haar measure $\omega_{\mu}$ on the orbit hypergroup $\Pi_{q, \mu}$ is given by the image measure of the (normalized) Lebesgue measure $(2 \pi)^{-p q d / 2} d x$ on $M_{p, q}$ under the mapping $x \mapsto|x|$. Using again polar coordinates, we obtain

$$
\omega_{\mu}(f)=\frac{2^{-\mu q}}{\Gamma_{\Omega_{q}}(\mu)} \int_{\Omega_{q}} f(\sqrt{r}) \Delta(r)^{\gamma} d r, \quad f \in C_{c}\left(\Pi_{q}\right) .
$$

Fourier transform and convolution of radial functions on $M_{p, q}$ are calculated with our notation a follows: suppose that $F, G \in L^{1}\left(M_{p, q}\right)$ are radial with $F(x)=f(|x|)$ and $G(x)=g(|x|)$. Then the Fourier transform of $F$ is

$$
\widehat{F}(\lambda)=\frac{1}{(2 \pi)^{d p q / 2}} \int_{M_{p, q}} F(x) e^{-i(\lambda \mid x)} d x=\int_{\Pi_{q}} f(r) \varphi_{|\lambda|}(r) d \omega_{\mu}(r)=\widehat{f}(|\lambda|) .
$$

The convolution of $F$ and $G$ is given by $F * G(x)=H(|x|)$ with

$$
\begin{aligned}
H(r) & =(2 \pi)^{-d p q / 2} \int_{M_{p, q}} F\left(\sigma_{0} r-y\right) G(y) d y \\
& =\frac{2^{-\mu q}}{\Gamma_{\Omega_{q}}(\mu)} \int_{\Omega_{q}}\left(\int_{\Sigma_{p, q}} f\left(\left|\sigma_{0} r-\sigma \sqrt{t}\right|\right) d \sigma\right) g(\sqrt{t}) \Delta(t)^{\gamma} d t \\
& =\int_{\Pi_{q}} f\left(r *_{\mu} s\right) g(s) d \omega_{\mu}(s)=\left(f *_{\mu} g\right)(r) .
\end{aligned}
$$

(Recall notation (2.5).) The multiplicativity of the characters $\varphi_{s}$ implies a positive product formula for the Bessel functions $\mathcal{J}_{\mu}$ with index $\mu=p d / 2, p \geqslant q$ an integer:

$$
\mathcal{J}_{\mu}\left(r^{2}\right) \mathcal{J}_{\mu}\left(s^{2}\right)=\int_{\Sigma_{p, q}} \mathcal{J}_{\mu}\left(r^{2}+s^{2}+r \widetilde{\sigma} s+s \widetilde{\sigma}^{*} r\right) d \sigma \quad \forall r, s \in \Pi_{q}
$$

We shall generalize this formula to Bessel functions of arbitrary index $\mu \in \mathbb{C}$ with $\mathfrak{R} \mu>d(q-1 / 2)$. For real indices we shall obtain a positive product formula, which leads to a continuous family of hypergroup structures on $\Pi_{q}$ beyond those which have a realization as orbit hypergroups as above. The decisive observation towards this aim is that only the reduced matrix $\widetilde{\sigma}$ is relevant in (3.5) and (3.2). In the following section, we introduce coordinates on the Stiefel manifold which are adapted to this situation.

\subsection{Split coordinates on the Stiefel manifold}

Let $k \in \mathbb{N}$ with $p-k \geqslant q$. We decompose $\sigma \in \Sigma_{p, q}$ as $\sigma=\left(\begin{array}{c}v \\ w\end{array}\right)$ with $v \in M_{k, q}$ and $w \in M_{p-k, q}$. For fixed $q$, put

$$
D_{k}=\left\{v \in M_{k, q}: v^{*} v<I\right\}
$$

where the notion $x<y$ for $x, y \in M_{q}(\mathbb{F})$ means that $y-x$ is (strictly) positive definite.

Proposition 3.2. The mapping

$$
\Phi: D_{k} \times \Sigma_{p-k, q} \longrightarrow \Sigma_{p, q}, \quad\left(v, \sigma^{\prime}\right) \longmapsto\left(\begin{array}{c}
v \\
\sigma^{\prime} \sqrt{I-v^{*} v}
\end{array}\right)
$$

is a diffeomorphism onto a dense and open subset $\widetilde{\Sigma}_{p, q}$ of $\Sigma_{p, q}$. Let $d \sigma$ and $d \sigma^{\prime}$ denote the normalized 


\section{BESSEL CONVOLUTIONS ON MATRIX CONES}

Riemannian volume elements on $\Sigma_{p, q}$ and $\Sigma_{p-k, q}$ respectively, and let $\eta:=\frac{d}{2}(p-k)-\frac{n}{q}$. Then on $\widetilde{\Sigma}_{p, q}$,

$$
d \sigma=c_{k, p} \cdot \Delta\left(I-v^{*} v\right)^{\eta} d \sigma^{\prime} d v
$$

where

$$
c_{k, p}=\left(\int_{D_{k}} \Delta\left(I-v^{*} v\right)^{\eta} d v\right)^{-1}=\pi^{-d k q / 2} \frac{\Gamma_{\Omega_{q}}(d p / 2)}{\Gamma_{\Omega_{q}}(d(p-k) / 2)} .
$$

In the important special case $p \geqslant 2 q, k=q$ with $\mathbb{F}=\mathbb{R}$, this result goes back to [Her55]. For the reader's convenience we nevertheless supply a concise proof along a different approach.

Proof. It is easily checked that $\Phi: D_{k} \times \Sigma_{p-k, q} \rightarrow \Sigma_{p, q}$ is $C^{1}$ and injective, and that its image $\widetilde{\Sigma}_{p, q}$ is dense in $\Sigma_{p, q}$. Let $f \in C\left(\Sigma_{p, q}\right)$ and extend it to $F \in L^{1}\left(M_{p, q}\right)$ by $F(\sigma \sqrt{r}):=f(\sigma)$ if $\frac{1}{2} I<r<\frac{3}{2} I$ and $F=0$ otherwise. Then

$$
\int_{M_{p, q}} F(x) d x=C \int_{\Sigma_{p, q}} f d \sigma \quad \text { with } C=C_{p, q} \cdot \int_{\frac{1}{2} I<r<\frac{3}{2} I} \Delta(r)^{\gamma} d r .
$$

On the other hand, we write $x \in M_{p, q}$ in block form as $x=\left(\begin{array}{l}x_{1} \\ x_{2}\end{array}\right)$ with $x_{1} \in M_{k, q}$ and $x_{2} \in M_{p-k, q}$. Then

$$
\int_{M_{p, q}} F(x) d x=C_{p-k, q} \int_{M_{k, q}} \int_{\Omega_{q}} \int_{\Sigma_{p-k, q}} F\left(\begin{array}{c}
x_{1} \\
\sigma_{2} \sqrt{r_{2}}
\end{array}\right) \Delta\left(r_{2}\right)^{\eta} d r_{2} d \sigma_{2} d x_{1}
$$

where we used polar coordinates $x_{2}=\sigma_{2} \sqrt{r_{2}}$ in $M_{p-k, q}$. Consider now the successive transforms $r_{2} \mapsto x_{1}^{*} x_{1}+r_{2}=: r$ with $x_{1}$ fixed and $x_{1} \mapsto v:=x_{1} r^{-1 / 2}$ with $r$ fixed. We have $d r_{2}=d r$ and, by [FT87, Lemma 2], $d x_{1}=\Delta(r)^{d k / 2} d v$. Thus, in a first step,

$$
\int_{M_{p, q}} F(x) d x=C_{p-k, q} \int_{M_{k, q}} \int_{x_{1}^{*} x_{1}+\Omega_{q}} \int_{\Sigma_{p-k, q}} F\left(\begin{array}{c}
x_{1} \\
\sigma_{2} \sqrt{r-x_{1}^{*} x_{1}}
\end{array}\right) \Delta\left(r-x_{1}^{*} x_{1}\right)^{\eta} d r d \sigma_{2} d x_{1}
$$

where

$$
F\left(\begin{array}{c}
x_{1} \\
\sigma_{2} \sqrt{r-x_{1}^{*} x_{1}}
\end{array}\right)=f\left(\begin{array}{c}
v \\
\sigma_{2} w
\end{array}\right) \text { with } w=\sqrt{r-\sqrt{r} v^{*} v \sqrt{r}} \cdot r^{-1 / 2}
$$

As $w^{*} w=I-v^{*} v$, it follows that $w=u \sqrt{I-v^{*} v}$ with some $u \in U_{q}$. The invariance of the Lebesgue measure on $M_{p-k, q}$ under the action of $U_{q}$ by right multiplication easily implies that $d \sigma_{2}$ is invariant under this action of $U_{q}$ on $\Sigma_{p-k, q}$. In view of the identity $\eta+k d / 2=\gamma$, the above integral therefore becomes

$$
\begin{aligned}
& C_{p-k, q} \int_{\frac{1}{2} I<r<\frac{3}{2} I} \int_{D_{k}} \int_{\Sigma_{p-k, q}} f\left(\begin{array}{c}
v \\
\sigma_{2} \sqrt{I-v^{*} v}
\end{array}\right) \Delta(r)^{\eta+k d / 2} \Delta\left(I-v^{*} v\right)^{\eta} d \sigma_{2} d v d r \\
& =C \cdot \frac{C_{p-k, q}}{C_{p, q}} \int_{D_{k}} \int_{\Sigma_{p-k, q}} f\left(\frac{v}{\sigma_{2} \sqrt{I-v^{*} v}}\right) \Delta\left(I-v^{*} v\right)^{\eta} d \sigma_{2} d v .
\end{aligned}
$$

Together with (3.6), this gives the stated Jacobian (which does not vanish on $D_{k} \times \Sigma_{p-k, q}$ ) and proves the claimed diffeomorphism property of $\Phi$.

If $p \geqslant 2 q$, then the above proposition with $k=q$ leads to a concise integration formula for functions $f(\sigma)$ on $\Sigma_{p, q}$ which depend only on the first $q$ rows of $\sigma$. For abbreviation, we put

$$
\varrho:=d\left(q-\frac{1}{2}\right)+1
$$

and for $\mu \in \mathbb{C}$ with $\Re \mu>\rho-1$,

$$
\kappa_{\mu}:=\int_{D_{q}} \Delta\left(I-v^{*} v\right)^{\mu-\rho} d v
$$




\section{RÖSLER}

The explicit value of $\kappa_{\mu}$ is obtained by using polar coordinates and [FK94, Theorem VII.1.7] about beta integrals on symmetric cones,

$$
\kappa_{\mu}=C_{q, q} \int_{r<I} \Delta(I-r)^{\mu-\varrho} \Delta(r)^{d / 2-1} d r=\pi^{d q^{2} / 2} \cdot \frac{\Gamma_{\Omega_{q}}(\mu-d q / 2)}{\Gamma_{\Omega_{q}}(\mu)} .
$$

Corollary 3.3. Let $p \geqslant 2 q$. Then for $f \in C\left(\Sigma_{p, q}\right)$ of the form

$$
f(\sigma)=\tilde{f}(\tilde{\sigma}), \quad \tilde{\sigma}=\sigma_{0}^{*} \sigma
$$

one has

$$
\int_{\Sigma_{p, q}} f d \sigma=\frac{1}{\kappa_{p d / 2}} \int_{D_{q}} \tilde{f}(v) \Delta\left(I-v^{*} v\right)^{p d / 2-\varrho} d v .
$$

The dependence on $p$ now occurs only in the density, not in the domain of integration.

\subsection{A product formula for Bessel functions with continuous index}

As the integrands in the convolution formula (3.2) and the product formula (3.5) depend only on the reduced matrix $\widetilde{\sigma}$, we obtain by Corollary 3.3 the following.

Proposition 3.4. Suppose that $p \geqslant 2 q$ and let $\mu=p d / 2$.

(1) The convolution $*_{\mu}$ on the orbit hypergroup $\Pi_{q, \mu}$ can be written as

$$
\left(\delta_{r} *_{\mu} \delta_{s}\right)(f)=\frac{1}{\kappa_{\mu}} \int_{D_{q}} f\left(\sqrt{r^{2}+s^{2}+r v s+s v^{*} r}\right) \Delta\left(I-v^{*} v\right)^{\mu-\varrho} d v .
$$

(2) The Bessel function $\mathcal{J}_{\mu}$ satisfies the product formula

$$
\mathcal{J}_{\mu}\left(r^{2}\right) \mathcal{J}_{\mu}\left(s^{2}\right)=\frac{1}{\kappa_{\mu}} \int_{D_{q}} \mathcal{J}_{\mu}\left(r^{2}+s^{2}+r v s+s v^{*} r\right) \Delta\left(I-v^{*} v\right)^{\mu-\varrho} d v \quad \forall r, s \in \Pi_{q} .
$$

We are now going to extend the integral formulas of Proposition 3.4 to arbitrary indices $\mu$ within the half plane $\left\{\mu \in \mathbb{C}: \mathfrak{R} \mu \supset-1=d\left(q-\frac{1}{2}\right)\right\}$. We use a standard technique (cf. [Ste88] for the rank-one case), namely analytic continuation with respect to $\mu$. The argumentation is based on a classical theorem of Carlson.

TheOREM 3.5 (Titchmarsh [Tit39, p. 186]). Let $f(z)$ be holomorphic in a neighbourhood of $\{z \in$ $\mathbb{C}: \mathfrak{R} z \geqslant 0\}$ satisfying $f(z)=O\left(e^{c|z|}\right)$ on $\mathfrak{R} z \geqslant 0$ for some $c<\pi$. If $f(z)=0$ for all $z \in \mathbb{N}_{0}$, then $f$ is identically zero.

The following theorem is the main result of this section.

Theorem 3.6. Let $\mu \in \mathbb{C}$ with $\Re \mu>\varrho-1=d\left(q-\frac{1}{2}\right)$. Then the Bessel function $\mathcal{J}_{\mu}$ satisfies the product formula

$$
\mathcal{J}_{\mu}\left(r^{2}\right) \mathcal{J}_{\mu}\left(s^{2}\right)=\frac{1}{\kappa_{\mu}} \int_{D_{q}} \mathcal{J}_{\mu}\left(r^{2}+s^{2}+r v s+s v^{*} r\right) \Delta\left(I-v^{*} v\right)^{\mu-\varrho} d v \quad \forall r, s \in \Pi_{q} .
$$

Remark. It is easy to obtain from (3.7) the more general identity

$$
\mathcal{J}_{\mu}\left(x^{*} x\right) \mathcal{J}_{\mu}\left(y^{*} y\right)=\frac{1}{\kappa_{\mu}} \int_{D_{q}} \mathcal{J}_{\mu}\left(x^{*} x+y^{*} y+x^{*} v y+y^{*} v^{*} x\right) \Delta\left(I-v^{*} v\right)^{\mu-\varrho} d v
$$

for arbitrary $x, y \in M_{q}$. For this, recall that every $x \in M_{q}$ has a polar decomposition $x=u|x|$ with $|x|=\sqrt{x^{*} x} \in \Pi_{q}$ and a unitary matrix $u \in U_{q}$. Choose $r=|x|=u^{*} x$ and $s=|y|=w^{*} y$ with $u, w \in U_{q}$ in (3.7). Then

$$
\mathcal{J}_{\mu}\left(x^{*} x\right) \mathcal{J}_{\mu}\left(y^{*} y\right)=\frac{1}{\kappa_{\mu}} \int_{D_{q}} \mathcal{J}_{\mu}\left(x^{*} x+y^{*} y+x^{*} u v w^{*} y+y^{*} w v^{*} u^{*} x\right) \Delta\left(I-v^{*} v\right)^{\mu-\varrho} d v .
$$




\section{BESSEL CONVOLUTIONS ON MATRIX CONES}

Under the coordinate transform $v \mapsto u v w^{*}=: \widetilde{v}$ we have $d v=d \widetilde{v}, \Delta\left(I-v^{*} v\right)=\Delta\left(I-\widetilde{v}^{*} \widetilde{v}\right)$ and $v \in D_{q} \Leftrightarrow \widetilde{v} \in D_{q}$. This implies (3.8).

Proof of Theorem 3.6. Let $W:=\{\mu \in \mathbb{C}: \Re \mu>\varrho-1\}$. By the asymptotic properties of the usual gamma function we have

$$
\kappa_{\mu} \sim\left(\frac{\pi}{\mu}\right)^{d q^{2} / 2} \quad \text { uniformly in } W \text { as } \mu \rightarrow \infty .
$$

Consider now the claimed product formula (3.7). Its left-hand side is holomorphic and, according to Corollary 2.2, also uniformly bounded in $W$ as a function of $\mu$. In order to estimate the right-hand side of (3.7), note that the argument $r^{2}+s^{2}+r v s+s v^{*} r$ is positive semidefinite for all $v \in D_{q}$. Moreover,

$$
\begin{aligned}
\operatorname{tr}\left(r^{2}+s^{2}+r v s+s v^{*} r\right) & =\|r\|^{2}+\|s\|^{2}+2(r \mid v s) \\
& \leqslant\|r\|^{2}+2\|r\|\|v s\|+\|s\|^{2} \leqslant(\|r\|+\|s\|)^{2}
\end{aligned}
$$

where again $\|\cdot\|$ is the Hilbert-Schmidt norm on $M_{q}$. For the last estimate, it was used that $I-v^{*} v$ is positive definite and therefore

$$
\|s\|^{2}-\|v s\|^{2}=\operatorname{tr}\left(s\left(I-v^{*} v\right) s\right) \geqslant 0 .
$$

Thus by Corollary 2.2,

$$
\left|\mathcal{J}_{\mu}\left(r^{2}+s^{2}+r v s+s v^{*} r\right)\right| \leqslant e^{(\|r\|+\|s\|)^{2}} \quad \forall v \in D_{q} .
$$

This easily implies that the right-hand side in (3.7) is also holomorphic as a function of $\mu$ in $W$, and can be estimated according to

$$
\left|\frac{1}{\kappa_{\mu}} \int_{D_{q}} \mathcal{J}_{\mu}\left(r^{2}+s^{2}+r v s+s v^{*} r\right) \Delta\left(I-v^{*} v\right)^{\mu-\varrho} d v\right| \leqslant C \cdot \frac{1}{\left|\kappa_{\mu}\right|} \cdot \int_{D_{q}} \Delta\left(I-v^{*} v\right)^{\Re \mu-\varrho} d v=C \cdot \frac{\kappa_{\Re \mu}}{\left|\kappa_{\mu}\right|}
$$

with a constant $C>0$ independent of $\mu$. In view of (3.9), the last expression is of the form

$$
O\left(|\mu|^{d q^{2} / 2}\right) \quad \text { as } \mu \rightarrow \infty \text { in } W .
$$

Now define $f(z):=g\left((z+2 q) \frac{d}{2}\right)$ where

$$
g(\mu):=\mathcal{J}_{\mu}\left(r^{2}\right) \mathcal{J}_{\mu}\left(s^{2}\right)-\frac{1}{\kappa_{\mu}} \int_{D_{q}} \mathcal{J}_{\mu}\left(r^{2}+s^{2}+r v s+s v^{*} r\right) \Delta\left(I-v^{*} v\right)^{\mu-\varrho} d v, \quad \mu \in W .
$$

Then $f(z)=0$ for all $z \in \mathbb{N}_{0}$ by Proposition 3.4. The above considerations further show that $f$ is holomorphic and of polynomial growth on $\mathfrak{R} z>-1$. With Carlson's theorem, the validity of (3.7) follows as claimed.

Remarks. (1) We may as well establish a Bochner-type integral representation for the Bessel function $J_{\mu}$ with $\Re \mu>\varrho-1$ by analytic continuation. Indeed, for $\mu=p d / 2$ with an integer $p \geqslant q$, we obtain from (3.4) that for all $x \in M_{q}$,

$$
\mathcal{J}_{\mu}\left(x^{*} x\right)=\int_{\Sigma_{p, q}} e^{-2 i\left(\sigma \mid \sigma_{0} x\right)} d \sigma=\int_{\Sigma_{p, q}} e^{-2 i(\widetilde{\sigma} \mid x)} d \sigma .
$$

If $p \geqslant 2 q$, then according to Corollary 3.3 this can be written as

$$
\mathcal{J}_{\mu}\left(x^{*} x\right)=\frac{1}{\kappa_{\mu}} \int_{D_{q}} e^{-2 i(v \mid x)} \Delta\left(I-v^{*} v\right)^{\mu-\varrho} d v .
$$

Analytic continuation with respect to $\mu$ as above shows that (3.12) remains valid for all $\mu \in \mathbb{C}$ with $\mathfrak{R} \mu>\varrho-1$. From this identity, it follows by the Riemann-Lebesgue lemma for the additive 


\section{RÖSLER}

matrix group $\left(M_{q},+\right)$ that $\mathcal{J}_{\mu} \in C_{0}\left(\Pi_{q}\right)$. In case $\mathbb{F}=\mathbb{R}$, the integral representation (3.12) for $\mathfrak{R} \mu>\varrho-1$ goes back to [Her55], where it was proven by a different method.

(2) In the rank-one case $q=1$ we have

$$
\mathcal{J}_{\mu}\left(\frac{r^{2}}{4}\right)=j_{\mu-1}(r) \quad\left(r \in \mathbb{R}_{+}\right)
$$

with the one-variable Bessel functions

$$
j_{\alpha}(z)={ }_{0} F_{1}\left(\alpha+1 ;-\frac{z^{2}}{4}\right)=\sum_{n=0}^{\infty} \frac{(-1)^{n} \Gamma(\alpha+1)}{n ! \Gamma(\alpha+n+1)}\left(\frac{z}{2}\right)^{2 n} .
$$

Formulas (3.12) and (3.7) reduce to the well-known Mehler formula

$$
j_{\alpha}(r)=c_{\alpha} \int_{-1}^{1} e^{-i r t}\left(1-t^{2}\right)^{\alpha-1 / 2} d t
$$

and the product formula

$$
j_{\alpha}(r) j_{\alpha}(s)=c_{\alpha} \int_{-1}^{1} j_{\alpha}\left(\sqrt{r^{2}+s^{2}+2 r s t}\right)\left(1-t^{2}\right)^{\alpha-1 / 2} d t,
$$

which are both valid for $\alpha \in \mathbb{C}$ with $\Re \alpha>-1 / 2$.

We finish this section with an alternative form of product formula (3.7) and the integral representation (3.12) for the Bessel functions $\mathcal{J}_{\mu}$. Let

$$
B:=\left\{z \in M_{1, q}(\mathbb{F}):|z|<1\right\}
$$

denote the unit ball in $M_{1, q}(\mathbb{F}) \cong \mathbb{F}^{q}$ with respect to the standard norm $|z|=\left(\sum_{j=1}^{q} \overline{z_{j}} z_{j}\right)^{1 / 2}$. It is easily checked that

$$
\Delta\left(I-z^{*} z\right)=1-|z|^{2}
$$

LEMma 3.7. The mapping

$$
P\left(y_{1}, \ldots, y_{q}\right):=\left(\begin{array}{c}
y_{1} \\
y_{2}\left(I-y_{1}^{*} y_{1}\right)^{1 / 2} \\
\vdots \\
y_{q}\left(I-y_{q-1}^{*} y_{q-1}\right)^{1 / 2} \cdots\left(I-y_{1}^{*} y_{1}\right)^{1 / 2}
\end{array}\right) ; \quad y_{1}, \ldots, y_{q} \in B
$$

establishes a diffeomorphism from $B^{q}$ onto $D_{q}$ with Jacobi determinant

$$
|\operatorname{det} d P(y)|=\prod_{j=1}^{q-1}\left(1-\left|y_{j}\right|^{2}\right)^{d(q-j) / 2} .
$$

Proof. For $k$ with $2 \leqslant k \leqslant q$, the mapping

$$
B \times D_{k-1} \longrightarrow D_{k}, \quad(z, w) \mapsto\left(\begin{array}{c}
z \\
w \sqrt{I-z^{*} z}
\end{array}\right)
$$

is obviously a diffeomorphism. By [FT87, Lemma 2] and Fubini's theorem, its Jacobi determinant is given by $\left(1-|z|^{2}\right)^{d(k-1) / 2}$. The $(q-1)$-fold iteration of this decomposition yields the assertion.

It is easily checked that for $v=P(y) \in D_{q}$,

$$
\Delta\left(I-v^{*} v\right)=\prod_{j=1}^{q} \Delta\left(I-y_{j}^{*} y_{j}\right)=\prod_{j=1}^{q}\left(1-\left|y_{j}\right|^{2}\right) .
$$

This implies the following corollary. 


\section{BESSEL CONVOLUTIONS ON MATRIX CONES}

Corollary 3.8. Let $\zeta \in \mathbb{C}$ with $\mathfrak{R} \zeta>-1$. Then the image measure of $\Delta\left(I-v^{*} v\right)^{\zeta} d v$ under $P^{-1}: D_{q} \rightarrow B^{q}$ is given by $\prod_{j=1}^{q}\left(1-\left|y_{j}\right|^{2}\right)^{\zeta+d(q-j) / 2} d y$.

Application to the product formula (3.7) and the integral representation (3.12) for the Bessel function $\mathcal{J}_{\mu}$ gives the following.

Corollary 3.9. Let $\mu \in \mathbb{C}$ with $\Re \mu>\varrho-1$. Then for all $r, s \in \Pi_{q}$,

$$
\begin{aligned}
\mathcal{J}_{\mu}\left(r^{2}\right) \mathcal{J}_{\mu}\left(s^{2}\right) & =\frac{1}{\kappa_{\mu}} \int_{B^{q}} \mathcal{J}_{\mu}\left(r^{2}+s^{2}+r P(y) s+s P(y)^{*} r\right) \prod_{j=1}^{q}\left(1-\left|y_{j}\right|^{2}\right)^{\mu-\varrho+d(q-j) / 2} d y \\
\mathcal{J}_{\mu}\left(r^{2}\right) & =\frac{1}{\kappa_{\mu}} \int_{B^{q}} e^{-2 i(P(y) \mid r)} \prod_{j=1}^{q}\left(1-\left|y_{j}\right|^{2}\right)^{\mu-\varrho+d(q-j) / 2} d y .
\end{aligned}
$$

\subsection{Bessel convolutions on the cone $\Pi_{q}$}

For real $\mu>\varrho-1$ the measure $\kappa_{\mu}^{-1} \cdot \Delta\left(I-v^{*} v\right)^{\mu-\varrho}$ in product formula (3.7) is a probability measure on $D_{q}$. We shall see that (3.7) leads to a hypergroup convolution on $\Pi_{q}$ which is of the same form as those of Proposition 3.4. This will give us a continuous series of commutative hypergroup structures on $\Pi_{q}$ which interpolate those occurring as orbit hypergroups for the indices $\mu=p d / 2, p \geqslant 2 q$ an integer.

Theorem 3.10. Fix an index $\mu \in \mathbb{R}$ with $\mu>\varrho-1$.

(a) The assignment

$$
\left(\delta_{r} *_{\mu} \delta_{s}\right)(f):=\frac{1}{\kappa_{\mu}} \int_{D_{q}} f\left(\sqrt{r^{2}+s^{2}+r v s+s v^{*} r}\right) \Delta\left(I-v^{*} v\right)^{\mu-\varrho} d v ; \quad f \in C\left(\Pi_{q}\right)
$$

defines a commutative hypergroup structure $\Pi_{q, \mu}=\left(\Pi_{q}, *_{\mu}\right)$ with neutral element 0 and the identity mapping as involution. The support of $\delta_{r} *_{\mu} \delta_{s}$ satisfies

$$
\operatorname{supp}\left(\delta_{r} *_{\mu} \delta_{s}\right) \subseteq\left\{t \in \Pi_{q}:\|t\| \leqslant\|r\|+\|s\|\right\} .
$$

(b) A Haar measure of the hypergroup $\Pi_{q, \mu}$ is given by

$$
\omega_{\mu}(f)=\frac{2^{-q \mu}}{\Gamma_{\Omega_{q}}(\mu)} \int_{\Omega_{q}} f(\sqrt{r}) \Delta(r)^{\gamma} d r
$$

with $\gamma=\mu-\frac{d}{2}(q-1)-1=\mu-\frac{n}{q}$.

Remark. The specific normalization of the Haar measure is motivated by Theorem 3.12 below.

Proof. (a) Clearly $\delta_{r} *_{\mu} \delta_{s}$ is a probability measure on $\Pi_{q}$ and

$$
\operatorname{supp}\left(\delta_{r} *_{\mu} \delta_{s}\right)=\left\{\sqrt{r^{2}+s^{2}+r v s+s v^{*} r}: v \in \overline{D_{q}}\right\},
$$

which does not depend on $\mu$. The stated support inclusion is immediate from the estimate (3.10). This shows hypergroup axiom (2). Property (3) is clear because it is known to be true for indices $\mu=p d / 2$ which lead to an orbit hypergroup structure. Property (4) is obvious, and (5) with $\bar{r}=r$ also follows in general because it is true in the orbit hypergroup cases. For the proof of (1) it suffices to show that for each $f \in C_{b}\left(\Pi_{q}\right)$ the mapping $(r, s) \mapsto f\left(r *_{\mu} s\right)$ is continuous. However, this is clear from the continuity of the map $(r, s, v) \mapsto f\left(\sqrt{r^{2}+s^{2}+r v s+s v^{*} r}\right)$ on $\Pi_{q}^{2} \times D_{q}$. Commutativity of the convolution is obvious, and for the proof of associativity it again suffices to consider point measures. So let $r, s, t \in \Pi_{q}$ and $f \in C_{b}\left(\Pi_{q}\right)$. Then

$$
\delta_{r} *_{\mu}\left(\delta_{s} *_{\mu} \delta_{t}\right)(f)=\frac{1}{\kappa_{\mu}^{2}} \int_{D_{q}} \int_{D_{q}} f(H(r, s, t ; v, w)) \Delta\left(I-v^{*} v\right)^{\mu-\varrho} \Delta\left(I-w^{*} w\right)^{\mu-\varrho} d v d w=: I(\mu)
$$




\section{RÖSLER}

with a certain $\Pi_{q}$-valued argument $H$ that is independent of the index $\mu$. Similarly,

$$
\left(\delta_{r} *_{\mu} \delta_{s}\right) *_{\mu} \delta_{t}(f)=I^{\prime}(\mu)
$$

with some $\mu$-independent argument $H^{\prime}$ instead of $H$. The integrals $I(\mu)$ and $I^{\prime}(\mu)$ are well-defined and holomorphic in $\{\mu \in \mathbb{C}: \mathfrak{R} \mu>\varrho-1\}$. Further, we know that $I(\mu)=I^{\prime}(\mu)$ for all $\mu=p d / 2$ with an integer $p \geqslant 2 q$. By analytic continuation as in the proof of Theorem 3.6 (use (3.9) again) we obtain validity of this relation for all $\mu$ with $\mathfrak{R} \mu>\varrho-1$.

(b) We need to prove that

$$
\int_{\Omega_{q}} f\left(s *_{\mu} \sqrt{r}\right) \Delta(r)^{\gamma} d r=\int_{\Omega_{q}} f(\sqrt{r}) \Delta(r)^{\gamma} d r
$$

for all $f \in C_{c}\left(\Pi_{q}\right)$ and $s \in \Pi_{q}$. Equivalently,

$$
\int_{\Omega_{q}} \int_{D_{q}} f\left(\sqrt{s^{2}+r+s v \sqrt{r}+\sqrt{r} v^{*} s}\right) \Delta\left(I-v^{*} v\right)^{\mu-\varrho} \Delta(r)^{\mu-n / q} d r d v=\int_{\Omega_{q}} f(\sqrt{r}) \Delta(r)^{\mu-n / q} d r .
$$

We know from $\S 3.1$ that this relation is true if $\mu=p d / 2$ with an integer $p \geqslant 2 q$. Moreover, the stated result is well known in the case $d=q=1$ for all $\mu$ in question; actually, in these cases $*_{\mu}$ is just the convolution of a Bessel-Kingman hypergroup (see [BH95, §3.5]). So we may assume that $d q \geqslant 2$. Again let $W=\{\mu \in \mathbb{C}: \Re \mu>\varrho-1\}$. Then $\mathfrak{R} \mu-n / q>0$ for all $\mu \in W$, and for fixed $f$ both sides of (3.13) are well-defined and holomorphic as functions of $\mu$ in $W$. We shall again carry out analytic continuation with respect to $\mu$, based on Carlson's theorem. We proceed in two steps.

Step 1. For $R>0$ let $B_{R}:=\left\{r \in \Pi_{q}:\|r\| \leqslant R\right\}$. Choose $R>0$ with $R^{q} \leqslant e^{\pi / d}$. The explicit formula for the convolution $*_{\mu}$ shows that there exist constants $S_{0}, R_{0}>0$ such that for $f \in C_{c}\left(\Pi_{q}\right)$ with supp $f \subseteq B_{R_{0}}$ and for $s \in \Pi_{q}$ with $\|s\| \leqslant S_{0}$ we have

$$
f\left(s *_{\mu} \sqrt{r}\right)=0 \quad \text { if }\|r\| \geqslant R .
$$

Suppose that $|f| \leqslant C$ on $\Pi_{q}$ and note that $\Delta(r) \leqslant\|r\|^{q}$. The left-hand side of (3.13) may then be estimated according to

$$
\begin{aligned}
|\mathrm{LHS}| & \leqslant C \int_{B_{R}} \Delta(r)^{\Re \mu-n / q} d r \cdot \int_{D_{q}} \Delta\left(I-v^{*} v\right)^{\Re \mu-\varrho} d v \\
& \leqslant C \cdot \kappa_{\Re \mu} \cdot \operatorname{vol}\left(B_{R}\right) \cdot\left(R^{q}\right)^{\Re \mu-n / q} .
\end{aligned}
$$

Due to the asymptotics (3.9), $\mu \mapsto \kappa_{\Re \mu}$ is bounded on $W$. Thus, by our initial assumption on $R$, we arrive at

$$
|\mathrm{LHS}|=O\left(e^{\pi / d|\mu|}\right) \quad \text { as } \mu \rightarrow \infty \text { in } W .
$$

The right-hand side of (3.13) coincides with the left-hand side for $s=0$ and is therefore of (at most) the same order as a function of $\mu$. As in the proof of Theorem 3.6, we have to substitute $\mu=(z+2 q) \frac{d}{2}$ to obtain holomorphic functions in $z$ on $\mathfrak{R} z>-1$ which coincide for all $z \in \mathbb{N}_{0}$ and are of order $O\left(e^{\pi|z| / 2}\right)$ in $\mathfrak{R} z \geqslant 0$. Carlson's theorem now implies the assertion under the above restrictions on $f$ and $s$.

Step 2. Let $f \in C_{c}\left(\Pi_{q}\right)$ and $s \in \Pi_{q}$ be arbitrary. For $\delta>0$ define $f_{\delta}(r):=f(\delta r)$. We shall use that the convolution $*_{\mu}$ is homothetic, i.e.

$$
f_{\delta}\left(r *_{\mu} s\right)=f\left(\delta r *_{\mu} \delta s\right)
$$




\section{BESSEL CONVOLUTIONS ON MATRIX CONES}

Fix constants $S_{0}, R_{0}$ as in step 1 and choose $\delta>1$ such that $\operatorname{supp} f_{\delta} \subseteq B_{R_{0}}$ and $\|s / \delta\| \leqslant S_{0}$. Then

$$
\begin{aligned}
I(s) & :=\int_{\Omega_{q}} f\left(s *_{\mu} \sqrt{r}\right) \Delta(r)^{\gamma} d r=\int_{\Omega_{q}} f_{\delta}\left(\frac{s}{\delta} *_{\mu} \frac{\sqrt{r}}{\delta}\right) \Delta(r)^{\gamma} d r \\
& =\delta^{2 q \gamma+2 n} \int_{\Omega_{q}} f_{\delta}\left(\frac{s}{\delta} *_{\mu} \sqrt{t}\right) \Delta(t)^{\gamma} d t .
\end{aligned}
$$

As $f_{\delta}$ and $s / \delta$ satisfy the conditions of step 1 , we conclude that $I(s)=I(0)$, which finishes the proof of part (b).

Our next aim is to determine the dual and the Plancherel measure of the hypergroup $\Pi_{q, \mu}$ with $\mu>\varrho-1$. For $s \in \Pi_{q}$ we define

$$
\varphi_{s}(r)=\varphi_{s}^{\mu}(r):=\mathcal{J}_{\mu}\left(\frac{1}{4} s r^{2} s\right), \quad r \in \Pi_{q} .
$$

Note that

$$
\varphi_{s}(r)=\varphi_{r}(s)
$$

because $J_{\mu}$ depends only on the eigenvalues of its argument. Moreover, we have the following.

Lemma 3.11. Let $\mu>\varrho-1$. Then for each $s \in \Pi_{q}, \varphi_{s}=\varphi_{s}^{\mu}$ belongs to $C_{b}\left(\Pi_{q}\right)$ with $\left\|\varphi_{s}\right\|_{\infty}=$ $\varphi_{s}(0)=1$. If $s$ belongs to the open cone $\Omega_{q}$, then even $\varphi_{s} \in C_{0}\left(\Pi_{q}\right)$.

Proof. The first assertion is immediate from the Bochner-type integral representation (3.12) for $\mathcal{J}_{\mu}$. Now suppose $s \in \Omega_{q}$. Then $r \rightarrow \infty$ in $\Pi_{q}$ implies that $r^{2} \rightarrow \infty$ and also $s r^{2} s \rightarrow \infty$, because $s$ is invertible. The second assertion thus follows from the fact that $\mathcal{J}_{\mu}$ vanishes at infinity.

From this lemma together with the product formula (3.8) it is immediate that each $\varphi_{s}$ with $s \in \Pi_{q}$ belongs to the dual $\widehat{\Pi}_{q, \mu}$. The results of $\S 3.1$ suggest that these Bessel functions actually make up the complete dual.

Theorem 3.12. (1) The dual space of $\Pi_{q, \mu}$ with $\mu>\rho-1$ is given by

$$
\widehat{\Pi}_{q, \mu}=\left\{\varphi_{s}=\varphi_{s}^{\mu}: s \in \Pi_{q}\right\} .
$$

(2) The hypergroup $\Pi_{q, \mu}$ is self-dual via the homeomorphism $\Psi: \Pi_{q, \mu} \rightarrow \widehat{\Pi}_{q, \mu}, s \mapsto \varphi_{s}$. Under this identification, the Plancherel measure $\pi_{\mu}$ on $\widehat{\Pi}_{q, \mu}$ coincides with the Haar measure $\omega_{\mu}$.

For the proof of part (1) we need the following.

Lemma 3.13. The set $A:=\left\{\varphi_{s}: s \in \Pi_{q}\right\}$ is closed in $\widehat{\Pi}_{q, \mu}$ with respect to the topology of locally uniform convergence.

Proof. Let $\left(\varphi_{s_{j}}\right)_{j \in \mathbb{N}}$ be a sequence in $A$ converging to $\alpha \in \widehat{\Pi}_{q, \mu}$ locally uniformly. If the sequence $\left(s_{j}\right) \subset \Pi_{q}$ is bounded, then after passing to a subsequence we may assume that $s_{j} \rightarrow s \in \Pi_{q}$ as $j \rightarrow \infty$. Then $\varphi_{s_{j}} \rightarrow \varphi_{s}$ and therefore $\alpha=\varphi_{s} \in A$. If, otherwise, the original sequence $\left(s_{j}\right)$ is unbounded, then after passing to a subsequence we may assume that $s_{j} \rightarrow \infty$. Thus, by Lemma 3.11, $\varphi_{s_{j}}(r)=\varphi_{r}\left(s_{j}\right) \rightarrow 0$ for all $r \in \Omega_{q}$ as $j \rightarrow \infty$. This implies $\alpha=0$ which contradicts the convention $0 \notin \widehat{\Pi}_{q, \mu}$.

Proof of Theorem 3.12. In a first step, we establish that $\Pi_{q, \mu}$ has subexponential growth in the sense of [Voi88], i.e. for each compact subset $K \subset \Pi_{q}$ and each $c>1$, the Haar measure satisfies $\omega_{\mu}\left(K^{m}\right)=o\left(c^{m}\right)$ as $m \rightarrow \infty$. Here $K^{m}$ denotes the $m$-fold convolution power of $K$, the convolution product of subsets $A, B$ of a hypergroup $(X, *)$ being defined by $A * B=\bigcup_{x \in A, y \in B} \operatorname{supp}\left(\delta_{x} * \delta_{y}\right)$. Once subexponential growth is known, [Voi88, Theorem 2.17] will imply that the support of the 


\section{RÖSLER}

Plancherel measure $\pi_{\mu}$ coincides with the complete dual $\widehat{\Pi}_{q, \mu}$. For the proof of subexponential growth, it suffices to consider the balls $B_{R}=\left\{r \in \Pi_{q}:\|r\| \leqslant R\right\}$. From the support properties of $*_{\mu}$ we see that $B_{R}^{m} \subseteq B_{m R}$. Moreover,

$$
\omega_{\mu}\left(B_{R}\right)=C \cdot \int_{\|\sqrt{r}\| \leqslant R} \Delta(r)^{\gamma} d r=O\left(R^{2 q \gamma+2 n}\right)=O\left(R^{2 q \mu}\right) \quad \text { as } R \rightarrow \infty .
$$

Thus, for fixed $R>0$, we obtain $\omega_{\mu}\left(B_{R}^{m}\right)=O\left(m^{2 q \mu}\right)$, and the assertion follows.

In a second step, we determine $\pi_{\mu}$. The decisive ingredient will be known results about the Hankel transform on a symmetric cone. For $s, r \in \Omega_{q}$, define

$$
H_{\mu}(s, r)=\frac{1}{\Gamma_{\Omega_{q}}(\mu)} \mathcal{J}_{\mu}(\sqrt{s} r \sqrt{s}) .
$$

Suppose that $\mu>d(q-1)+1$. Then according to [FK94, Theorem XV.4.1], the Hankel transform

$$
U_{\mu} F(s):=\int_{\Omega_{q}} H_{\mu}(s, r) F(r) \Delta(r)^{\gamma} d r
$$

defines an isometric and involutive isomorphism of $L^{2}\left(\Omega_{q}, \Delta(r)^{\gamma} d r\right)$. The argumentation of [Her55] and $[\mathrm{FT} 87, \S 5]$ shows that this statement actually extends to all $\mu \in \mathbb{R}$ with $\mu>\frac{d}{2}(q-1)$, i.e. $\gamma>-1$. Let $f \in L^{2}\left(\Pi_{q}, \omega_{\mu}\right)$. Then $F(r):=f(\sqrt{r})$ belongs to $L^{2}\left(\Omega_{q}, \Delta(r)^{\gamma} d r\right)$ and a short calculation shows that

$$
\widehat{f}\left(\varphi_{s}\right)=\int_{\Pi_{q}} \varphi_{s}(r) f(r) d \omega_{\mu}(r)=2^{-q \mu} U_{\mu} F\left(\frac{s^{2}}{4}\right) \quad \forall s \in \Omega_{q} .
$$

Moreover, by the isometry of $U_{\mu}$ we readily obtain

$$
\int_{\Pi_{q}}\left|\widehat{f}\left(\varphi_{s}\right)\right|^{2} d \omega_{\mu}(s)=\int_{\Pi_{q}}|f(s)|^{2} d \omega_{\mu}(s) .
$$

This shows that the Plancherel measure $\pi_{\mu}$ associated with $\omega_{\mu}$ is given by

$$
\pi_{\mu}(g)=\int_{\Pi_{q}} g\left(\varphi_{s}\right) d \omega_{\mu}(s), \quad g \in C_{c}\left(\widehat{\Pi}_{q, \mu}\right) .
$$

Hence, the support of $\pi_{\mu}$, which we already know to coincide with $\widehat{\Pi}_{q, \mu}$, also coincides with the closure of the set $\left\{\varphi_{s}: s \in \Pi_{q}\right\}$ in $\widehat{\Pi}_{q, \mu}$ with respect to the topology of locally uniform convergence. The proof of part (1) is therefore accomplished by Lemma 3.13. For part (2), it remains to verify that $\Psi$ is a homeomorphism. Continuity and surjectivity are clear. For injectivity, suppose $\varphi_{s}=\varphi_{r}$. Then in view of (3.15) we have $\widehat{\delta}_{s}=\widehat{\delta}_{r}$ and the injectivity of the Fourier transform of measures on the hypergroup $\Pi_{q, \mu}$ implies $s=r$. To check continuity of $\Psi^{-1}$ suppose that $\varphi_{s_{i}} \rightarrow \varphi_{s}$ locally uniformly. Then $\widehat{\delta}_{s_{i}} \rightarrow \widehat{\delta}_{s}$ locally uniformly on $\widehat{\Pi}_{q, \mu}$. Levy's continuity theorem [BH95, Theorem 4.2.2] implies that $\delta_{s_{i}} \rightarrow \delta_{s}$ weakly, and hence $s_{i} \rightarrow s$.

\subsection{The limit case $\mu=\varrho-1$}

Using Corollary 3.8 we see that the convolution $*_{\mu}$ with $\mu>\varrho-1$ can be written in the alternative form

$$
\left(\delta_{r} *_{\mu} \delta_{s}\right)(f)=\frac{1}{\kappa_{\mu}} \int_{B^{q}} f\left(\sqrt{r^{2}+s^{2}+r P(y) s+s P(y)^{*} r}\right) \prod_{j=1}^{q}\left(1-\left|y_{j}\right|^{2}\right)^{\mu-\varrho+\frac{d}{2}(q-j)} d y .
$$

We shall use this representation to determine the limit of the convolution $*_{\mu}$ as $\mu \downarrow \rho-1$, where it assumes a degenerate form. As $\rho-1=p d / 2$ with $p=2 q-1$, it is natural to expect that the resulting limit convolution coincides with the orbit hypergroup convolution $*_{\rho-1}$ on $\Pi_{q}$ derived from $M_{q, 2 q-1}$. 


\section{Bessel CONVOLUTIONS ON MATRIX CONES}

In the following, $d \sigma$ denotes the normalized surface measure on the unit sphere $S=\left\{z \in M_{1, q}\right.$ : $|z|=1\}$. The coordinate transform $P: B^{q} \rightarrow D_{q}$ of Lemma 3.7 is assumed to be continuously extended to $\bar{B}^{q}$.

Proposition 3.14. As $\mu \downarrow \varrho-1$, the convolution product $\delta_{r} *_{\mu} \delta_{s}$ converges weakly to the probability measure $\delta_{r} \tilde{*} \delta_{s}$ on $\Pi_{q}$ given by

$$
\begin{aligned}
& \left(\delta_{r}^{\sim} * \delta_{s}\right)(f) \\
& \quad=\widetilde{\kappa} \int_{B^{q-1}} \int_{S} f\left(\sqrt{r^{2}+s^{2}+r P(y) s+s P(y)^{*} r}\right) \prod_{j=1}^{q-1}\left(1-\left|y_{j}\right|^{2}\right)^{\mu-\varrho+\frac{d}{2}(q-j)} d y_{1} \cdots d y_{q-1} d \sigma\left(y_{q}\right)
\end{aligned}
$$

with a normalization constant $\widetilde{\kappa}>0$. The product $\tilde{*}$ defines a commutative hypergroup structure on $\Pi_{q}$ which coincides with the orbit hypergroup $\Pi_{q}^{\rho-1}$ derived from $M_{q, 2 q-1}$ as in $\S 3.1$. In particular,

$$
\left(\delta_{r}{ }^{\sim} * \delta_{s}\right)(f)=\left(\delta_{r} *_{\rho-1} \delta_{s}\right)(f)=\int_{\Sigma_{q, 2 q-1}} f\left(\sqrt{r^{2}+s^{2}+r \widetilde{\sigma} s+(r \widetilde{\sigma} s)^{*}}\right) d \sigma
$$

and the additional statements of Theorems 3.10 and 3.12 extend to the case $\mu=\varrho-1$.

Proof. For $\mu>\varrho-1$ consider the probability measure

$$
p_{\mu}:=c_{\mu}^{-1}\left(1-|y|^{2}\right)^{\mu-\varrho} 1_{B}(y) d y
$$

on $M_{1, q}$, where $c_{\mu}:=\int_{B}\left(1-|y|^{2}\right)^{\mu-\varrho} d y$ and $1_{B}$ denotes the characteristic function of the ball $B$. It is easily checked that $p_{\mu}$ tends weakly to the normalized surface measure $d \sigma$ on $S$ as $\mu \rightarrow \varrho-1$. Indeed, let $f \in C\left(M_{1, q}\right)$ and put $F(\tau):=\int_{S} f(\tau y) d \sigma(y), \tau \geqslant 0$. Then with $c_{\mu}^{\prime}=\int_{0}^{1}\left(1-\tau^{2}\right)^{\mu-\varrho} \tau^{d q-1} d \tau$,

$$
\int_{B} f d p_{\mu}=\frac{1}{c_{\mu}^{\prime}} \int_{0}^{1} F(\tau)\left(1-\tau^{2}\right)^{\mu-\varrho} \tau^{d q-1} d \tau \longrightarrow F(1) \quad \text { as } \mu \rightarrow \varrho-1 .
$$

This proves that $\delta_{r} *_{\mu} \delta_{s} \rightarrow \delta_{r} \tilde{*} \delta_{s}$ weakly. It is clear that $\operatorname{supp}\left(\delta_{r} \tilde{*} \delta_{s}\right) \subseteq \operatorname{supp}\left(\delta_{r} *_{\mu} \delta_{s}\right)$ for $\mu>\varrho-1$ which implies the same support inclusion as in Theorem 3.10(a). In the limit $\mu \rightarrow \varrho-1$ we further obtain that the Bessel functions

$$
\varphi_{s}(r)=\mathcal{J}_{\varrho-1}\left(\frac{1}{4} s r^{2} s\right), \quad s, r \in \Pi_{q}
$$

satisfy the product formula

$$
\varphi_{s}(r) \varphi_{s}(t)=\int_{\Pi_{q}} \varphi_{s}(\tau) d\left(\delta_{r}^{\sim} * \delta_{t}\right)(\tau) \quad \forall s \in \Pi_{q}
$$

On the other hand, consider the orbit hypergroup $X_{\varrho-1}=\left(\Pi_{q}, *_{\varrho-1}\right)$ derived from $M_{p, q}$ with $p=2 q-1$. Its dual space consists exactly of the Bessel functions $\varphi_{s}(r)=\mathcal{J}_{\rho-1}\left(\frac{1}{4} r s^{2} r\right)$ as above. In particular,

$$
\varphi_{s}(r) \varphi_{s}(t)=\int_{\Pi_{q}} \varphi_{s}(\tau) d\left(\delta_{r} *_{\varrho-1} \delta_{t}\right)(\tau) \quad \forall s \in \Pi_{q}
$$

The injectivity of the Fourier transform on the hypergroup $X_{\varrho-1}$ now implies that $\delta_{r} *_{\varrho-1} \delta_{t}=\delta_{r} \tilde{*} \delta_{t}$ for all $r, t$.

Remarks. (1) We conjecture that the formulas of Corollary 3.9 permit degenerate extensions to successively larger index ranges. As soon as the exponent in one of the iterated integrals becomes critical, the corresponding integral over $B$ should be replaced by an integral over $S$. More precisely, we conjecture that within the range

$$
\left\{\mu \in \mathbb{R}: \varrho-\frac{d}{2}(q-k)-1<\mu \leqslant \varrho-\frac{d}{2}(q-k-1)-1\right\}, \quad k=q-1, \ldots, 1
$$




\section{RÖSLER}

the following product formula is valid:

$$
\begin{aligned}
\mathcal{J}_{\mu}\left(r^{2}\right) \mathcal{J}_{\mu}\left(s^{2}\right)= & \frac{1}{\kappa_{\mu, j}} \int_{B^{k}} \int_{S^{q-k}} \mathcal{J}_{\mu}\left(r^{2}+s^{2}+r P(y) s+s P(y)^{*} r\right) \\
& \times \prod_{j=1}^{k}\left(1-\left|y_{j}\right|^{2}\right)^{\mu-\varrho+\frac{d}{2}(q-j)} d y_{1} \ldots d y_{k} d \sigma\left(y_{k+1}\right) \ldots d \sigma\left(y_{q}\right) .
\end{aligned}
$$

Also, there should be analogous integral representations for $\mathcal{J}_{\mu}$ within the above ranges of $\mu$.

(2) Further properties of the hypergroups $\Pi_{q, \mu}$ concerning their automorphism groups as well as stochastic aspects (such as limit theorems for random walks on matrix cones associated with Bessel convolutions) have recently been studied in [Voi06].

\section{Hypergroups associated with rational Dunkl operators of type $B_{q}$}

In the analysis of the previous sections, one may be interested in questions which depend only on the spectra of the matrices from the underlying cone $\Pi_{q}=\Pi_{q}(\mathbb{F})$. This amounts to considering functions and measures on $\Pi_{q}$ which are invariant under unitary conjugation. For $x \in H_{q}$ we denote by $\sigma(x)=\left(\xi_{1}, \ldots, \xi_{q}\right) \in \mathbb{R}^{q}$ the set of eigenvalues of $x$ ordered by size, i.e. $\xi_{1} \geqslant \cdots \geqslant \xi_{q}$. The unitary group $U_{q}$ acts on $\Pi_{q}$ via conjugation, $(u, r) \mapsto u r u^{-1}$. The orbits under this action are parametrized by the set $\Xi_{q}$ of possible spectra $\sigma(r)$ of matrices $r \in \Pi_{q}$,

$$
\Xi_{q}=\left\{\xi=\left(\xi_{1}, \ldots, \xi_{q}\right) \in \mathbb{R}^{q}: \xi_{1} \geqslant \cdots \geqslant \xi_{q} \geqslant 0\right\} .
$$

Recall at this point that $U_{q}$ is the maximal compact subgroup of the automorphism group of $\Omega_{q}$; the latter coincides with $\mathrm{GL}_{q}=\mathrm{GL}_{q}(\mathbb{F})$, acting on $H_{q}$ via $(g, x) \mapsto g x g^{*}$. The set $\Xi_{q}$ is a closed Weyl chamber of the hyperoctahedral group $B_{q}=S_{q} \ltimes \mathbb{Z}_{2}^{q}$ which acts on $\mathbb{R}^{q}$ by permutations of the basis vectors and sign changes. In $\S 3$ we saw that $\Pi_{q}$ carries a continuously parametrized family of commutative hypergroup structures $*_{\mu}$ with $\mu \geqslant \varrho-1$, as well as additional orbit hypergroup structures for $\mu=p d / 2, p \geqslant q$ an integer. In the following section we are going to show that under the above action of $U_{q}$ on $\Pi_{q}$ each convolution $*_{\mu}$ induces a commutative orbit hypergroup convolution $\circ_{\mu}$ on $\Xi_{q}$, in a similar manner as the orbit hypergroups were obtained from group convolutions in $\S 2.3$. In $\S 4.2$ we then identify the characters of $\left(\Xi_{q}, \circ_{\mu}\right)$ with multivariable Bessel functions of Dunkl type which are associated with the root system of type $B_{q}$. In effect, we thus obtain a continuous series of commutative hypergroup structures on the chamber $\Xi_{q}$ whose characters are given by Dunkl-type Bessel functions.

\subsection{Convolutions on the spectra of positive definite matrices}

In the situation described above, the canonical mapping

$$
\pi: \Pi_{q} \rightarrow \Xi_{q}, \quad r \mapsto \sigma(r)
$$

is continuous, surjective, and open with respect to the standard topologies on both sets. Therefore, the $\operatorname{map} \Pi_{q}^{U_{q}} \rightarrow \Xi_{q}, U_{q} \cdot r \mapsto \sigma(r)$ becomes a homeomorphism when $\Pi_{q}^{U_{q}}$ is equipped with the quotient topology. In the following, $d u$ denotes the normalized Haar measure on $U_{q}$ and $\xi \in \Xi_{q}$ is always identified with the diagonal matrix $\operatorname{diag}\left(\xi_{1}, \ldots, \xi_{q}\right) \in \Pi_{q}$ without mentioning. Moreover, we introduce the index set

$$
\left.\mathcal{M}_{q}:=\left\{\frac{p d}{2}, p=q, q+1, \ldots\right\} \cup\right] \rho-1, \infty[
$$




\section{BESSEL CONVOLUTIONS ON MATRIX CONES}

Theorem 4.1. (1) For each $\mu \in \mathcal{M}_{q}$ the chamber $\Xi_{q}$ carries a commutative hypergroup structure with convolution

$$
\left(\delta_{\xi} \circ_{\mu} \delta_{\eta}\right)(f):=\int_{U_{q}}(f \circ \pi)\left(\xi *_{\mu} u \eta u^{-1}\right) d u, \quad f \in C\left(\Xi_{q}\right) .
$$

The neutral element of the hypergroup $\Xi_{q, \mu}:=\left(\Xi_{q}, \circ_{\mu}\right)$ is $0 \in \Xi_{q}$ and the involution is given by the identity mapping.

(2) A Haar measure on $\Xi_{q, \mu}$ is given by

$$
\widetilde{\omega}_{\mu}=\pi\left(\omega_{\mu}\right)=d_{\mu} h_{\mu}(\xi) d \xi \quad \text { with } h_{\mu}(\xi)=\prod_{i=1}^{q} \xi_{i}^{2 \gamma+1} \prod_{i<j}\left(\xi_{i}^{2}-\xi_{j}^{2}\right)^{d}
$$

and a constant $d_{\mu}>0$.

Remarks. (1) The constant $d_{\mu}$ is determined in $\S 4.2$.

(2) In the generic case $\mu>\varrho-1$ the convolution $\circ_{\mu}$ can be more explicitly written as

$$
\left(\delta_{\xi} \circ_{\mu} \delta_{\eta}\right)(f)=\frac{1}{\kappa_{\mu}} \int_{D_{q}} \int_{U_{q}} f\left(\sigma\left(\sqrt{\xi^{2}+u \eta^{2} u^{-1}+\xi v u \eta u^{-1}+u \eta u^{-1} v^{*} \xi}\right)\right) \Delta\left(I-v^{*} v\right)^{\mu-\varrho} d u d v .
$$

We start with some preparations for the proof of Theorem 4.1.

Lemma 4.2. For $u \in U_{q}$ consider the homeomorphism of $\Pi_{q}$ given by $T_{u}: r \mapsto u r u^{-1}$. The image measure of the convolution product $\delta_{r} *_{\mu} \delta_{s} \in M^{1}\left(\Pi_{q}\right)$ under $T_{u}$ is given by

$$
T_{u}\left(\delta_{r} *_{\mu} \delta_{s}\right)=\delta_{u r u^{-1}} *_{\mu} \delta_{u s u^{-1}} .
$$

Proof. Recall that the measure $\Delta\left(I-v^{*} v\right)^{\mu-\varrho} d v$ in the convolution formula of Theorem 3.10 is invariant under unitary conjugation. This yields immediately that

$$
\left(g \circ T_{u}\right)\left(r *_{\mu} s\right)=g\left(u r u^{-1} *_{\mu} u s u^{-1}\right) \quad \forall g \in C\left(\Pi_{q}\right) .
$$

Aside. This lemma just says that $T_{u}$ is actually a hypergroup automorphism for each of the convolutions $*_{\mu}$. The next lemma is a special case of [FK94, Theorem VI.2.3].

Lemma 4.3. For integrable functions $g: \Pi_{q} \rightarrow \mathbb{C}$,

$$
\int_{\Pi_{q}} g(r) d r=\kappa \int_{\Xi_{q}} \int_{U_{q}} g\left(u \xi u^{-1}\right) d u \prod_{i<j}\left(\xi_{i}-\xi_{j}\right)^{d} d \xi
$$

with a normalization constant $\kappa=\kappa_{q}>0$.

Proof of Theorem 4.1. (1) We employ the technique of [Jew75, § 13] for the transfer of hypergroup structures via orbital mappings. Note first that the continuous open surjection $\pi: \Pi_{q} \rightarrow \Xi_{q}$ is also proper (because $U_{q}$ is compact) and thus provides an orbital mapping from the hypergroup $\Pi_{q, \mu}$ onto $\Xi_{q}$ in the sense of $[\operatorname{Jew} 75, \S 13]$. For $\xi \in \Xi_{q}$ define

$$
\epsilon_{\xi}:=\int_{U_{q}} \delta_{u \xi u^{-1}} d u
$$

which is a probability measure on $\Pi_{q}$ and satisfies $\operatorname{supp} \epsilon_{\xi}=\pi^{-1}(\xi)$. We claim that each $\epsilon_{\xi}$ is $\pi$-consistent in the following sense:

$$
\pi\left(\epsilon_{\xi} *_{\mu} \delta_{s}\right)=\pi\left(\epsilon_{\xi} *_{\mu} \delta_{t}\right) \quad \text { for all } s, t \in \Pi_{q} \text { with } \pi(s)=\pi(t)
$$




\section{RÖSLER}

here $\pi$ is extended to $M^{+}\left(\Pi_{q}\right)$ by taking image measures. For the proof of (4.1), suppose that $s, t \in \Pi_{q}$ satisfy $\pi(s)=\pi(t)$. Then for each $g \in C\left(\Pi_{q}\right)$ which is invariant under $U_{q}$-conjugation we have

$$
\int_{\Pi_{q}} g d\left(\epsilon_{\xi} *_{\mu} \delta_{s}\right)=\int_{U_{q}} g\left(u \xi u^{-1} *_{\mu} s\right) d u=\int_{U_{q}} g\left(\xi *_{\mu} u^{-1} s u\right) d u
$$

Note that Lemma 4.2 has been used for the second identity. As $s$ and $t$ have the same spectra, the last integral does not change when $s$ is replaced by $t$. This proves (4.1). The orbital mapping $\pi$ also satisfies $\pi(0)=0 \in \Xi_{q}$ and $\pi^{-1}(0)=0 \in \Pi_{q}$. We can now apply [Jew75, Theorem 13.5.A]. This shows that $\Xi_{q}$ becomes a commutative hypergroup with convolution

$$
\delta_{\xi} \circ_{\mu} \delta_{\eta}=\pi\left(\epsilon_{\xi} *_{\mu} \epsilon_{\eta}\right),
$$

the identity mapping as involution and neutral element $\pi(0)=0$. This proves the assertions of part (1).

(2) According to [Jew75, Theorem 13.3.A], a Haar measure $\widetilde{\omega}_{\mu}$ on $\Xi_{q, \mu}$ is given by the image measure of $\omega_{\mu}$ under $\pi$. Let $f \in C_{c}\left(\Xi_{q}\right)$ and put $g=f \circ \pi$, which is $U_{q}$-invariant. Then

$$
\int_{\Xi_{q}} f d \widetilde{\omega}_{\mu}=\kappa \int_{\Pi_{q}} g d \omega_{\mu}=\kappa^{\prime} \int_{\Xi_{q}} g(\sqrt{\xi}) \prod_{i=1}^{q} \xi_{i}^{\gamma} \prod_{i<j}\left(\xi_{i}-\xi_{j}\right)^{d} d \xi
$$

where $\sqrt{\xi}=\left(\sqrt{\xi_{1}}, \ldots, \sqrt{\xi_{q}}\right)$. Up to a constant factor, the last integral coincides with $\int_{\Xi_{q}} g(\xi)$ $h_{\mu}(\xi) d \xi$.

Remarks. (1) Recall that for $\mu=p d / 2$ with an integer $p \geqslant q$ the hypergroup $\Pi_{q, \mu}$ is just the orbit hypergroup obtained from the multiplication action of the unitary group $U_{p}$ on $M_{p, q}$. In this case, the above hypergroup structure of $\left(\Xi_{q}, \circ_{\mu}\right)$ can also be described as an orbit hypergroup derived directly from $M_{p, q}$, as follows. Consider the action of the group $L:=U_{p} \times U_{q}$ on $M_{p, q}$ by

$$
x \mapsto u x v^{-1}, \quad(u, v) \in L .
$$

The orbits of this action are parametrized by the possible sets of singular values of matrices from $M_{p, q}$. Indeed, let $\sigma_{\text {sing }}(x)=\sigma\left(\sqrt{x^{*} x}\right)=\left(\xi_{1}, \ldots, \xi_{q}\right) \in \Xi_{q}$ denote the singular spectrum of $x \in M_{p, q}$, the singular values being ordered by size. We have the equivalences

$$
\begin{aligned}
\sigma_{\text {sing }}(x)=\sigma_{\text {sing }}(y) & \Longleftrightarrow \sigma\left(x^{*} x\right)=\sigma\left(y^{*} y\right) \\
& \Longleftrightarrow \exists v \in U_{q}: y^{*} y=v x^{*} x v^{-1}=\left(x v^{-1}\right)^{*}\left(x v^{-1}\right) \\
& \Longleftrightarrow \exists(u, v) \in U_{p} \times U_{q}: y=u x v^{-1} .
\end{aligned}
$$

Therefore the orbit space $M_{p, q}^{L}$ can be identified with the chamber $\Xi_{q}$ via $U_{p} x U_{q} \mapsto \sigma_{\text {sing }}(x)$, and this is easily checked to be a homeomorphism with respect to the natural topologies on both spaces. Note that $\left(u x v^{-1}\right)^{*} u x v^{-1}=v x^{*} x v^{-1}$. Hence, under the mapping $\Phi: M_{p, q} \rightarrow \Pi_{q}, x \mapsto \sqrt{x^{*} x}$, the above action of $L$ on $M_{p, q}$ induces the conjugation action of $U_{q}$ on $\Pi_{q}$. Moreover, the orbit convolution on $\Pi_{q, \mu}$ is defined in such a way that

$$
\delta_{r} *_{\mu} \delta_{s}=\Phi\left(Q_{r} * Q_{s}\right) \quad \text { with } Q_{r}=\int_{U_{p}} \delta_{u \sigma_{0} r} d u \in M^{1}\left(M_{p, q}\right)
$$

where $*$ denotes the usual convolution on the additive group $M_{p, q}$. This shows that for $\mu=p d / 2$, the convolution of the hypergroup $\Xi_{q, \mu}$ coincides with the convolution of the orbit hypergroup $M_{p, q}^{L} \cong \Xi_{q}$, which is, in turn, naturally identified with the convolution of the Gelfand pair $\left(L \ltimes M_{p, q}, L\right)$.

(2) For $\mu=p d / 2$ with an integer $p \geqslant q$, the support of the measure $\delta_{\xi} \circ_{\mu} \delta_{\eta}$ describes the set of possible singular spectra of sums $x+y$ made up by matrices $x, y \in M_{p, q}(\mathbb{F})$ with given singular spectra $\xi$ and $\eta$. 


\section{BESSEL CONVOLUTIONS ON MATRIX CONES}

Let us return to general indices $\mu \in \mathcal{M}_{q}$. In analogy to Lemma 2.6 for orbit hypergroups from groups, we expect that the characters of the hypergroup $\Xi_{q, \mu}$ are all obtained by taking $U_{q}$-means of the characters of $\Pi_{q, \mu}$. For $\xi \in \Xi_{q}$, define $\psi_{\xi}=\psi_{\xi}^{\mu} \in C_{b}\left(\Xi_{q}\right)$ by

$$
\psi_{\xi}(\eta):=\int_{U_{q}} \varphi_{\xi}\left(u \eta u^{-1}\right) d u
$$

where $\varphi_{\xi}(r)=\varphi_{\xi}^{\mu}(r)=\mathcal{J}_{\mu}\left(\frac{1}{4} r \xi^{2} r\right)$. The $\varphi_{\xi}$ are the characters of $\Pi_{q, \mu}$ which are parametrized by diagonal matrices. Note that by $U_{q}$-invariance of $\mathcal{J}_{\mu}$ we have

$$
\varphi_{s}\left(u r u^{-1}\right)=\varphi_{u^{-1}} s u(r) \quad \forall r, s \in \Pi_{q} .
$$

Hence, the mean of $\varphi_{s}$ is equal to the mean of $\varphi_{\xi}$ for $\xi=\sigma(s)$ and $\psi_{\xi}(\eta)=\psi_{\eta}(\xi)$ for all $\xi, \eta \in \Xi_{q}$.

TheOREM 4.4. Let $\mu \in \mathcal{M}_{q}$.

(1) The dual space of the hypergroup $\Xi_{q, \mu}=\left(\Xi_{q}, \circ_{\mu}\right)$ is given by

$$
\widehat{\Xi}_{q, \mu}=\left\{\psi_{\xi}=\psi_{\xi}^{\mu}: \xi \in \Xi_{q}\right\} .
$$

(2) The hypergroup $\Xi_{q, \mu}$ is self-dual via the homeomorphism $\Xi_{q, \mu} \rightarrow \widehat{\Xi}_{q, \mu}, \xi \mapsto \psi_{\xi}$. Under this identification, the Plancherel measure $\widetilde{\pi}_{\mu}$ of $\Xi_{q, \mu}$ coincides with the Haar measure $\widetilde{\omega}_{\mu}$.

Proof. (1) It is easily checked that each $\psi_{\xi}$ is multiplicative with respect to $\circ_{\mu}$ and therefore belongs to $\widehat{\Xi}_{q, \mu}$. Indeed, for $\eta, \zeta \in \Xi_{q}$ we calculate

$$
\psi_{\xi}\left(\eta \circ_{\mu} \zeta\right)=\int_{U_{q}}\left(\psi_{\xi} \circ \pi\right)\left(\eta *_{\mu} u \zeta u^{-1}\right) d u=\int_{\Pi_{q}} \int_{U_{q}} \int_{U_{q}} \varphi_{\xi}\left(v r v^{-1}\right) d\left(\delta_{\eta} *_{\mu} \delta_{u \zeta u^{-1}}\right)(r) d u d v .
$$

By Lemma 4.2 this is equal to

$$
\int_{U_{q}} \int_{U_{q}} \varphi_{\xi}\left(v \eta v^{-1} *_{\mu} v u \zeta u^{-1} v^{-1}\right) d u d v=\psi_{\xi}(\eta) \psi_{\xi}(\zeta) .
$$

It remains to show that each character of $\Xi_{q, \mu}$ is of the form $\psi_{\xi}$ with some $\xi \in \Xi_{q}$. For this, note first that the hypergroup $\Xi_{q, \mu}$ has subexponential growth, just as $\Pi_{q, \mu}$. Thus according to [Voi88, Theorem 2.17], the support of the Plancherel measure $\widetilde{\pi}_{\mu}$ of $\Xi_{q, \mu}$ coincides with the full dual $\widehat{\Xi}_{q, \mu}$. Let $\psi \in \widehat{\Xi}_{q, \mu}=\operatorname{supp} \widetilde{\pi}_{\mu}$. Then by [Voi91, Corollary 6] there exists a sequence of functions $f_{n} \in C_{c}\left(\Xi_{q}\right)$ such that $f_{n} \circ{ }_{\mu} \bar{f}_{n}$ converges to $\psi$ locally uniformly. Hence, $\left(f_{n} \circ \pi\right) *_{\mu}\left(\bar{f}_{n} \circ \pi\right)$ converges to $\psi \circ \pi$ locally uniformly on $\Pi_{q}$, which implies that $\psi \circ \pi$ is positive definite on the hypergroup $\Pi_{q, \mu}$. Note that $(\psi \circ \pi)(0)=1$. Thus, by Bochner's theorem for commutative hypergroups [Jew75, Theorem 12.3.B] and the self-duality of $\Pi_{q, \mu}$, there exists a probability measure $a \in M^{1}\left(\Pi_{q}\right)$ such that

$$
(\psi \circ \pi)(r)=\int_{\Pi_{q}} \varphi_{s}(r) d a(s) \quad \forall r \in \Pi_{q} .
$$

As $\psi \circ \pi$ is invariant under the action of $U_{q}$ and in view of (4.2), the measure $a$ must be $U_{q}$-invariant as well, i.e. $\int f\left(u r u^{-1}\right) d a(r)=\int f(r) d a(r)$ for all $f \in C_{c}\left(\Pi_{q}\right)$. Let $\widetilde{a}$ denote the image measure of $a$ under $\pi$. Then with $\eta=\pi(r)$ relation (4.3) becomes

$$
\psi(\eta)=\int_{\Pi_{q}} \varphi_{s}(\eta) d a(s)=\int_{\Xi_{q}} \psi_{\xi}(\eta) d \widetilde{a}(\xi) .
$$

On the other hand, by Bochner's theorem for the hypergroup $\Xi_{q, \mu}$, the character $\psi$ of $\Xi_{q, \mu}$ is an extremal point of the set of positive definite functions $f$ on the hypergroup $\Xi_{q, \mu}$ with the additional property $f(0)=1$. This implies that $\widetilde{a}$ must be a point measure, i.e. $\widetilde{a}=\delta_{\xi}$ for some $\xi \in \Xi_{q}$. Hence, $\psi=\psi_{\xi}$ 


\section{RÖSLER}

(2) The self-duality of $\Xi_{q, \mu}$ is proven in the same way as that of $\Pi_{q, \mu}$ (Theorem 3.12). To determine the Plancherel measure, let $f \in C_{c}\left(\Xi_{q}\right)$ and $g:=f \circ \pi$. Then the Fourier transforms of $f$ and $g$ with respect to the hypergroup structures $\Xi_{q, \mu}$ and $\Pi_{q, \mu}$ are related via

$$
\widehat{g}(s)=\int_{\Pi_{q}} g(r) \varphi_{s}(r) d \omega_{\mu}(r)=\int_{\Xi_{q}} f(\xi) \psi_{\sigma(s)}(\xi) d \widetilde{\omega}_{\mu}(\xi)=(\widehat{f} \circ \pi)(s) .
$$

By the Plancherel theorem for $\Pi_{q, \mu}$ we readily obtain $\int_{\Xi_{q}}|f|^{2} d \widetilde{\omega}_{\mu}=\int_{\Xi_{q}}|\widehat{f}|^{2} d \widetilde{\omega}_{\mu}$. This finishes the proof.

We would like to write the characters $\psi_{\xi}$ in a more explicit form. Recall that $\varphi_{s}(r)=\mathcal{J}_{\mu}\left(\frac{1}{4} s r^{2} s\right)$ for $r, s \in \Pi_{q}$, where $\mathcal{J}_{\mu}$ is given in terms of the spherical series (2.2). The spherical polynomials satisfy the product formula

$$
\frac{Z_{\lambda}(r) Z_{\lambda}(s)}{Z_{\lambda}(I)}=\int_{U_{q}} Z_{\lambda}\left(\sqrt{r} u s u^{-1} \sqrt{r}\right) d u \quad \forall r, s \in \Pi_{q},
$$

see [FK94, Corollary XI.3.2] or [GR87, Proposition 5.5]. This implies an integral representation for the Bessel functions $\mathcal{J}_{\mu}$ of two matrix arguments (recall $\S 2.2$ ):

$$
\mathcal{J}_{\mu}(r, s)=\int_{U_{q}} \mathcal{J}_{\mu}\left(\sqrt{r} u s u^{-1} \sqrt{r}\right) d u, \quad r, s \in \Pi_{q} .
$$

Thus, for $\xi, \eta \in \Xi_{q}$ we have

$$
\psi_{\xi}(\eta)=\int_{U_{q}} \mathcal{J}_{\mu}\left(\frac{1}{4} \xi u \eta^{2} u^{-1} \xi\right) d u=\mathcal{J}_{\mu}\left(\frac{\xi^{2}}{2}, \frac{\eta^{2}}{2}\right) .
$$

We shall use this representation in order to identify the characters $\psi_{\xi}$ with Dunkl-type Bessel functions for the root system of type $B_{q}$.

\subsection{Bessel functions associated with root systems}

Bessel functions associated with root systems are an important ingredient in the theory of rational Dunkl operators, which was initiated by Dunkl in the late 1980s [Dun89, Dun91]. They are a symmetrized version of the Dunkl kernel, which is the analogue of the usual exponential function in this theory. As a subclass, they include the spherical functions of a Cartan motion group, cf. [deJ06]. In this section we give a brief account on Dunkl theory and the associated Bessel functions; for a general background, the reader is referred to [DX01], [Opd93] and [Rös03b].

Let $G$ be a finite reflection group on $\mathbb{R}^{q}$ (equipped with the usual Euclidean scalar product $\langle\cdot, \cdot\rangle)$, and let $R$ be the reduced root system of $G$. We extend the action of $G$ to $\mathbb{C}^{q}$ and $\langle\cdot, \cdot\rangle$ to a bilinear form on $\mathbb{C}^{q} \times \mathbb{C}^{q}$. A function $k: R \rightarrow \mathbb{C}$ which is invariant under $G$ is called a multiplicity function on $R$. Important special cases of reflection groups are the symmetric group $S_{q}$ which acts on $\mathbb{R}^{q}$ by permuting the standard basis vectors $e_{i}$, and the hyperoctahedral group $B_{q}=S_{q} \ltimes \mathbb{Z}_{2}^{q}$ which acts by permutations of the basis vectors and sign changes. The root system of $B_{q}$ is given by $R=\left\{ \pm e_{i}, 1 \leqslant i \leqslant q\right\} \cup\left\{ \pm e_{i} \pm e_{j}, 1 \leqslant i<j \leqslant q\right\}$, and a multiplicity on it is of the form $k=\left(k_{1}, k_{2}\right)$ where $k_{1}$ is the value on the roots $\pm e_{i}$ and $k_{2}$ is the value on the roots $\pm e_{i} \pm e_{j}$.

For a finite reflection group $G$ and a fixed multiplicity function $k$ on its root system, the associated (rational) Dunkl operators are defined by

$$
T_{\xi}=T_{\xi}(k)=\partial_{\xi}+\frac{1}{2} \sum_{\alpha \in R} k_{\alpha}\langle\alpha, \xi\rangle \frac{1}{\langle\alpha, \cdot\rangle}\left(1-\sigma_{\alpha}\right), \quad \xi \in \mathbb{C}^{q} ;
$$

here $\sigma_{\alpha}$ denotes the reflection in the hyperplane perpendicular to $\alpha$ and the action of $G$ is extended to functions on $\mathbb{C}^{q}$ via $g . f(\xi)=f\left(g^{-1} \xi\right)$. The $T_{\xi}$ are homogeneous of degree -1 on the space 


\section{BESSEL CONVOLUTIONS ON MATRIX CONES}

$\mathcal{P}=\mathbb{C}\left[\mathbb{C}^{q}\right]$ of polynomial functions on $\mathbb{C}^{q}$ and they commute: $T_{\xi} T_{\eta}=T_{\eta} T_{\xi}$ (see [Dun89]). Hence, the map $\xi \mapsto T_{\xi}$ extends uniquely to a linear map $p \mapsto p(T), \mathcal{P} \rightarrow \operatorname{End}\left(\mathbb{C}^{q}\right)$. The Dunkl operators induce a sesquilinear pairing

$$
[p, q]_{k}=(p(T) \bar{q})(0)
$$

on $\mathcal{P}$, where $\bar{q}(\xi):=\overline{q(\bar{\xi})}$. In the following we assume that $k$ is non-negative. Then $[p, q]_{k}$ is actually a scalar product on $\mathcal{P}$, see [DO03, Proposition 2.4]. Moreover, for each fixed $w \in \mathbb{C}^{q}$, the joint eigenvalue problem

$$
T_{\xi} f=\langle\xi, w\rangle f \quad \forall \xi \in \mathbb{C}^{q} ; \quad f(0)=1
$$

has a unique holomorphic solution $f(z)=E_{k}(z, w)$ called the Dunkl kernel. It is symmetric in its arguments and satisfies $E_{k}(\lambda z, w)=E_{k}(z, \lambda w)$ for all $\lambda \in \mathbb{C}$ as well as $E_{k}(g z, w)=E_{k}(z, g w)$ for all $g \in G$. The generalized Bessel function

$$
J_{k}(z, w):=\frac{1}{|G|} \sum_{g \in G} E_{k}(z, g w)
$$

is $G$-invariant in both arguments. Moreover, $g(z)=J_{k}(z, w)$ is the unique holomorphic solution of the 'Bessel system'

$$
p(T) g=p(w) g \quad \forall p \in \mathcal{P}^{G} ; \quad g(0)=1
$$

where $\mathcal{P}^{G}$ denotes the subalgebra of $G$-invariant polynomials in $\mathcal{P}$, see [Opd93]. For crystallographic reflection groups and certain values of $k$, the operators $p(T)$, when restricted to $G$-invariant functions on $\mathbb{R}^{q}$, constitute the system of invariant differential operators of a Euclidean-type symmetric space and the Bessel functions $J_{k}(\cdot, w)$ can be identified with the associated spherical functions; for details see [deJ06]. The Dunkl kernel $E_{k}$ gives rise to an integral transform on $\mathbb{R}^{q}$ called the Dunkl transform. Let $w_{k}$ denote the weight function

$$
w_{k}(x)=\prod_{\alpha \in R}|\langle\alpha, x\rangle|^{2 k_{\alpha}}
$$

on $\mathbb{R}^{q}$. The Dunkl transform is the integral transform on $L^{1}\left(\mathbb{R}^{q}, w_{k}\right)$ defined by

$$
f \mapsto \widehat{f}^{k}, \quad \widehat{f}^{k}(\xi)=c_{k}^{-1} \int_{\mathbb{R}^{q}} f(x) E_{k}(-i \xi, x) w_{k}(x) d x \quad\left(\xi \in \mathbb{R}^{q}\right)
$$

with the constant

$$
c_{k}:=\int_{\mathbb{R}^{q}} e^{-|x|^{2} / 2} w_{k}(x) d x .
$$

A thorough study of this transform is given in [deJ93]. It has many properties in common with the usual Fourier transform to which it reduces in the case $k=0$. In particular, the Dunkl transform (as normalized above) extends to an isometric isomorphism of $L^{2}\left(\mathbb{R}^{q}, w_{k}\right)$, and $\left(T_{\eta} f\right)^{\wedge k}(\xi)=i\langle\xi, \eta\rangle \widehat{f}^{k}(\xi)$ for differentiable $f$ of sufficient decay. It is a long-standing open question whether $L^{1}\left(\mathbb{R}^{q}, w_{k}\right)$ can be given the structure of a commutative Banach algebra so that the Dunkl transform becomes the Gelfand transform on its (symmetric) spectrum, in a similar manner as for commutative hypergroups. In the rank-one case there is such a convolution, but it is not positivity-preserving. For details and affirmative results in this direction see [Rös03a]. It is, however, conjectured that for arbitrary $G$ and $k \geqslant 0$, the Bessel functions $J_{k}$ have a positive product formula which leads to a commutative hypergroup structure on a distinguished closed Weyl chamber $\Xi$ of $G$, the dual of this hypergroup being made up by the functions $\xi \mapsto J_{k}(\xi, \eta), \eta \in \Xi$. In rank one and in all Cartan motion group cases this is true, see [Rös03a]. In the following, we confirm this conjecture for three continuous series of multiplicities for root system $B_{q}$. Indeed, we identify the characters of the hypergroups $\Xi_{q, \mu}$ in $\S 4.1$ with Dunkl-type Bessel functions for $B_{q}$ and thus obtain hypergroup structures with these Bessel functions as characters. 


\section{RÖSLER}

\subsection{Dunkl theory and the convolutions on the Weyl chamber}

Let us denote by $J_{k}^{B}$ the Dunkl-type Bessel function associated with the reflection group $G=B_{q}$ and multiplicity $k=\left(k_{1}, k_{2}\right)$, and by $[\cdot, \cdot]_{k}^{B}$ the associated Dunkl pairing. For $z=\left(z_{1}, \ldots, z_{q}\right) \in \mathbb{C}^{q}$ we put $z^{2}=\left(z_{1}^{2}, \ldots, z_{q}^{2}\right)$. The following key result identifies $J_{k}^{B}$ with a generalized ${ }_{0} F_{1}$-hypergeometric function of two arguments (recall the notions of $\S 2.2$ ). It goes essentially back to [BF97, $\S 6]$, but the reasoning there is rather sketchy and there is an erraneous sign in one of the arguments. We therefore include a proof by different methods.

Proposition 4.5. Let $k=\left(k_{1}, k_{2}\right) \geqslant 0$ and $k_{2}>0$. Then for all $z, w \in \mathbb{C}^{q}$,

$$
J_{k}^{B}(z, w)={ }_{0} F_{1}^{\alpha}\left(\mu ; \frac{z^{2}}{2}, \frac{w^{2}}{2}\right) \text { with } \alpha=\frac{1}{k_{2}}, \mu=k_{1}+(q-1) k_{2}+\frac{1}{2} .
$$

Proof. The modified Jack polynomials $p_{\lambda}(z)=C_{\lambda}^{\alpha}\left(z^{2}\right)$, indexed by partitions $\lambda \geqslant 0$, are homogeneous of degree $2|\lambda|$ and form a basis of the vector space $\mathcal{P}^{G}$ for $G=B_{q}$. Thus, the Bessel function $J_{k}^{B}$ has a homogeneous expansion of the form

$$
J_{k}^{B}(z, w)=\sum_{\lambda \geqslant 0} a_{\lambda}(w) p_{\lambda}(z)
$$

with certain coefficients $a_{\lambda}(w) \in \mathbb{C}$. In view of the Bessel system we have

$$
\left.p_{\lambda}\left(T^{z}\right) J_{k}^{B}(z, w)\right|_{z=0}=p_{\lambda}(w) J_{k}^{B}(0, w)=C_{\lambda}^{\alpha}\left(w^{2}\right),
$$

where the superscript $z$ indicates operation with respect to the variable $z$. On the other hand, the results of [BF97] (relation (2.9) and the formula on top of p. 214) imply that the $p_{\lambda}$ are orthogonal with respect to $[\cdot, \cdot]_{k}^{B}$ with

$$
\left[p_{\lambda}, p_{\lambda}\right]_{k}^{B}=4^{|\lambda|}|\lambda| !(\mu)_{\lambda}^{\alpha} \cdot C_{\lambda}^{\alpha}(\mathbf{1})=: M_{\lambda}, \quad \mu=k_{1}+(q-1) k_{2}+\frac{1}{2} .
$$

Differentiation of (4.6) (recall that $p_{\nu}$ has real coefficients) now gives

$$
\left.p_{\lambda}\left(T^{z}\right) J_{k}^{B}(z, w)\right|_{z=0}=\sum_{\nu \geqslant 0} a_{\lambda}(w)\left[p_{\lambda}, p_{\nu}\right]_{k}^{B}=M_{\lambda} a_{\lambda}(w) .
$$

Hence, $a_{\lambda}(w)=M_{\lambda}^{-1} C_{\lambda}^{\alpha}\left(w^{2}\right)$, which implies the assertion.

As a consequence of (2.4), Bessel functions associated with a symmetric cone can now be identified with Dunkl Bessel functions of type $B_{q}$ with specific multiplicities.

Corollary 4.6. Let $\Omega$ be an irreducible symmetric cone inside a Euclidean Jordan algebra of rank $q$. Then for $r, s \in \bar{\Omega}$ with eigenvalues $\xi=\left(\xi_{1}, \ldots, \xi_{q}\right)$ and $\eta=\left(\eta_{1}, \ldots, \eta_{q}\right)$, respectively, we have

$$
\mathcal{J}_{\mu}\left(\frac{r^{2}}{2}, \frac{s^{2}}{2}\right)=J_{k}^{B}(\xi, i \eta)
$$

where $k$ is given by $k=k(\mu, d)=\left(\mu-\frac{d}{2}(q-1)-\frac{1}{2}, \frac{d}{2}\right)$.

Now consider again the hypergroup structures $\Xi_{q, \mu}=\left(\Xi_{q}, \circ_{\mu}\right)$ on

$$
\Xi_{q}=\left\{\xi=\left(\xi_{1}, \ldots, \xi_{q}\right) \in \mathbb{R}^{q}: \xi_{1} \geqslant \cdots \geqslant \xi_{q} \geqslant 0\right\}
$$

which is actually a closed Weyl chamber for the reflection group $B_{q}$. The consequence of our above identification can be formulated in a twofold way.

Corollary 4.7. The characters of the hypergroup $\Xi_{q, \mu}, \mu \in \mathcal{M}_{q}$ are given by

$$
\psi_{\eta}(\xi)=J_{k}^{B}(\xi, i \eta), \quad \eta \in \Xi_{q}
$$

with the multiplicity $k=k(\mu, d)$ as in the previous corollary. 


\section{Bessel CONVOLUTIONS ON MATRIX CONES}

COROllary 4.8. Consider the root system of type $B_{q}$ with a multiplicity $k=\left(k_{1}, k_{2}\right)$ where $k_{2}=\frac{d}{2}$ with $d \in\{1,2,4\}$ and $k_{1}=\frac{d}{2}(p-q+1)-\frac{1}{2}$ for integer $p \geqslant q$ or arbitrary $k_{1} \geqslant \frac{1}{2}(d q-1)$. Then the associated Dunkl-type Bessel functions $\xi \mapsto J_{k}^{B}(\xi, i \eta)$ are the characters of the hypergroup $\left(\Xi_{q}, \circ_{\mu}\right)$ on the closed Weyl chamber $\Xi_{q}$, where $\mu=k_{1}+(q-1) k_{2}+\frac{1}{2}$ and the convolution $\circ_{\mu}$ is defined over $\mathbb{F}=\mathbb{R}, \mathbb{C}, \mathbb{H}$, depending on the value of $d$. In particular, the Bessel function $J_{k}^{B}$ satisfies the positive product formula

$$
J_{k}^{B}(\xi, z) J_{k}^{B}(\eta, z)=\int_{\Xi_{q}} J_{k}^{B}(\zeta, z) d\left(\delta_{\xi} \circ_{\mu} \delta_{\eta}\right)(\zeta) \quad \forall \xi, \eta \in \Xi_{q}, z \in \mathbb{C}^{q} .
$$

The hypergroup Fourier transform on $\Xi_{q, \mu}$ is given by

$$
\widehat{f}(\eta)=\int_{\Xi_{q}} f(\xi) J_{k}^{B}(\xi, i \eta) d \widetilde{\omega}_{\mu}(\xi),
$$

with $\widetilde{\omega}_{\mu}=d_{\mu} h_{\mu}(\xi) d \xi$, as in Theorem 4.1. Note that $h_{\mu}$ coincides up to a constant factor with the weight $w_{k}$ for $k=k(\mu, d)$. As $w_{k}$ is $B_{q}$-invariant, we therefore have

$$
\widehat{f}(\eta)=\operatorname{constant} \cdot \widehat{F}^{k}(\eta),
$$

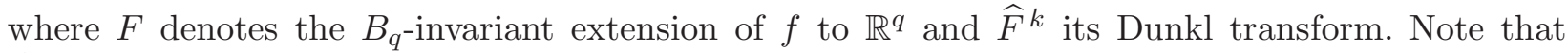
$\widehat{F}^{k}$ is $B_{q}$-invariant as well. With the Plancherel theorem for the Dunkl transform at hand, we are now also in a position to determine the normalization constant $d_{\mu}$ of $\widetilde{\omega}_{\mu}$ as announced previously. Indeed, recall from Theorem 4.4 that the Plancherel measure of the hypergroup $\Xi_{q, \mu}$ coincides with $\widetilde{\omega}_{\mu}$ under the natural identification of $\Xi_{q, \mu}$ with its dual. Using this and the Plancherel theorem for the Dunkl transform, we readily obtain

$$
\widehat{f}=\left.\widehat{F}^{k}\right|_{\Xi_{q}}
$$

and

$$
d_{\mu}=\left(\int_{\Xi_{q}} h_{\mu}(x) e^{-|x|^{2} / 2} d x\right)^{-1} .
$$

The value of $d_{\mu}$ can be calculated explicitly; it is a particular case of a Selberg type integral which was evaluated by Macdonald [Mac82] for the classical root systems.

In the general Dunkl setting, there is a generalized translation on suitable function spaces which replaces the usual group addition to some extent, see [Rös98, Rös03a] and the references cited there. On $L^{2}\left(\mathbb{R}^{q}, w_{k}\right)$, this translation is defined by

$$
\tau_{\eta} f(\xi)=c_{k}^{-1} \int_{\mathbb{R}^{q}} \widehat{f}^{k}(\xi) E_{k}(i \xi, \zeta) E_{k}(i \eta, \zeta) w_{k}(\zeta) d \zeta
$$

One has $\tau_{\eta}: L^{2}\left(\mathbb{R}^{q}, w_{k}\right) \rightarrow L^{2}\left(\mathbb{R}^{q}, w_{k}\right)$ with $\left(\tau_{\eta} f\right)^{\wedge k}(\zeta)=E_{k}(i \eta, \zeta) \hat{f}^{k}(\zeta)$. If we restrict to the Weyl group invariant case for $B_{q}$ with multiplicities as in Corollary 4.8, then this generalized translation just coincides with the translation defined in terms of hypergroup convolution. If, say, $f$ belongs to $L^{2}\left(\mathbb{R}^{q}, w_{k}\right)$ and is also continuous and Weyl group invariant, then with the notions of Corollary 4.8 we have

$$
\tau_{\eta} f(\xi)=\delta_{\xi} *_{\mu} \delta_{\eta}(f) \quad \forall \xi, \eta \in \Xi_{q} .
$$

When $\mu=p d / 2$ with an integer $p \geqslant q$, i.e. $k_{1}=\frac{d}{2}(p-q+1)-\frac{1}{2}$, then the Bessel functions $J_{k}^{B}(\cdot, z)$ can be identified with the spherical functions of the Cartan motion group associated with the Grassmann manifold $U(p, q) /\left(U_{p} \times U_{q}\right)$. This follows from the discussion in [deJ06] (see also [Rös03a]), and is in accordance with Remark (1) after the proof of Theorem 4.1 which implies that for $\mu=p d / 2$, the hypergroup convolution of $\Xi_{q, \mu}$ coincides with that of biinvariant measures for the Gelfand pair $\left(\left(U_{p} \times U_{q}\right) \ltimes M_{p, q}, U_{p} \times U_{q}\right)$. The multiplicative functions coincide with the 


\section{RÖSLER}

(elementary) spherical functions of this Gelfand pair. Thus, the hypergroups $\Xi_{q, \mu}$ with $\mu \geqslant \rho-1$ interpolate the discrete series of convolution algebras derived from the tangent space analysis on Grassmann manifolds.

\section{ACKNOWLEDGEMENTS}

The paper was for the most part written while the author was staying at the Korteweg-de Vries Institute for Mathematics at the University of Amsterdam, being supported by the Netherlands Organisation for Scientific Research (NWO). It is a pleasure to thank the Korteweg-de Vries Institute at the University of Amsterdam for their kind hospitality. Also, the support of SFB/TR-12 at the University of Bochum is greatly acknowledged. Special thanks go to Tom Koornwinder and to Michael Voit for various helpful comments and discussions.

\section{REFERENCES}

BF97 T. H. Baker and P. J. Forrester, The Calogero-Sutherland model and generalized classical polynomials, Comm. Math. Phys. 188 (1997), 175-216.

BH95 W. R. Bloom and H. Heyer, Harmonic analysis of probability measures on hypergroups, De Gruyter Studies in Mathematics, vol. 20 (de Gruyter, Berlin, 1995).

BO93 R. J. Beerends and E. M. Opdam, Certain hypergeometric series related to the root system BC, Trans. Amer. Math. Soc. 339 (1993), 581-609.

Con63 A. G. Constantine, Some non-central distribution problems in multivariate analysis, Ann. Math. Statist. 34 (1963), 1270-1285.

Dib90 H. Dib, Fonctions de Bessel sur une algébre de Jordan, J. Math. Pures Appl. (9) 69 (1990), 403-448.

Dun89 C. F. Dunkl, Differential-difference operators associated to reflection groups, Trans. Amer. Math. Soc. 311 (1989), 167-183.

Dun91 C. F. Dunkl, Integral kernels with reflection group invariance, Canad. J. Math. 43 (1991), 1213-1227.

DO03 C. F. Dunkl and E. M. Opdam, Dunkl operators for complex reflection groups, Proc. London Math. Soc. (3) 86 (2003), 70-108.

DX01 C. F. Dunkl and Y. Xu, Orthogonal polynomials of several variables (Cambridge University Press, Cambridge, 2001).

deJ93 M. F. E. de Jeu, The Dunkl transform, Invent. Math. 113 (1993), 147-162.

deJ06 M. F. E. de Jeu, Paley-Wiener theorems for the Dunkl transform, Trans. Amer. Math. Soc. 358 (2006), 4225-4250.

Far92 J. Faraut, Prolongement analytique des series de Taylor spheriques, in Hypergeometric functions on domains of positivity, Jack polynomials, and applications, Tampa, FL, 1991, Contemporary Mathematics, vol. 138 (American Mathematical Society, Providence, RI, 1992), 139-149.

FK94 J. Faraut and A. Korányi, Analysis on symmetric cones, Oxford Mathematical Monographs (Clarendon Press, Oxford, 1994).

FT87 J. Faraut and G. Travaglini, Bessel functions associated with representations of formally real Jordan algebras, J. Funct. Anal. 71 (1987), 123-141.

GR87 K. Gross and D. Richards, Special functions of matrix argument. I: Algebraic induction, zonal polynomials, and hypergeometric functions, Trans. Amer. Math. Soc. 301 (1987), 781-811.

GR89 K. Gross and D. Richards, Total positivity, spherical series, and hypergeometric functions of matrix argument, J. Approx. Theory 59 (1989), 224-246.

Her55 C. S. Herz, Bessel functions of matrix argument, Ann. of Math. (2) 61 (1955), 474-523.

Jam75 A. T. James, Special functions of matrix and single argument in statistis, in Proceedings of Theory and Application of Special Functions, University of Wisconsin, Madison, WI, 1975, ed. R. Askey (Academic Press, New York, 1975). 


\section{BESSEL CONVOLUTIONS ON MATRIX CONES}

Jew75 R. I. Jewett, Spaces with an abstract convolution of measures, Adv. Math. 18 (1975), 1-101.

Kan93 J. Kaneko, Selberg integrals and hypergeometric functions associated with Jack polynomials, SIAM J. Math. Anal. 24 (1993), 1086-1100.

Kin63 J. F. C. Kingman, Random walks with spherical symmetry, Acta Math. 109 (1963), 11-53.

KS97 F. Knop and S. Sahi, A recursion and combinatorial formula for Jack polynomials, Invent. Math. 128 (1997), 9-22. (Constr. Approx. 13 (1997), 537-567.)

Mac82 I. G. Macdonald, Some conjectures for root systems, SIAM J. Math. Anal. 13 (1982), 988-1007.

Mac87 I. G. Macdonald, Commuting differential operators and zonal spherical functions, in Algebraic groups, Utrecht, 1986, eds A. M. Cohen et al., Lecture Notes in Mathematics, vol. 1271 (Springer, Berlin, 1987).

Mui82 R. J. Muirhead, Aspects of multivariate statistical theory (John Wiley \& Sons, New York, 1982).

Opd93 E. M. Opdam, Dunkl operators, Bessel functions and the discriminant of a finite Coxeter group, Compositio Math. 85 (1993), 333-373.

Rös98 M. Rösler, Generalized Hermite polynomials and the heat equation for Dunkl operators, Comm. Math. Phys. 192 (1998), 519-542.

Rös03a M. Rösler, A positive radial product formula for the Dunkl kernel, Trans. Amer. Math. Soc. 355 (2003), 2413-2438.

Rös03b M. Rösler, Dunkl operators: theory and applications, in Orthogonal polynomials and special functions, Leuven, 2002, Lecture Notes in Mathematics, vol. 1817 (Springer, Berlin, 2003), 93-135.

Ros78 K. Ross, Centers of hypergroups, Trans. Amer. Math. Soc. 243 (1978), 251-269.

Sta89 R. P. Stanley, Some combinatorial properties of Jack symmetric functions, Adv. Math. 77 (1989), 76-115.

Ste88 K. Stempak, A new proof of Sonine's formula, Proc. Amer. Math. Soc. 104 (1988), 453-457.

Tit39 E. C. Titchmarsh, The theory of functions (Oxford University Press, Oxford, 1939).

Voi88 M. Voit, Positive characters on commutative hypergroups and some applications, Math. Z. 198 (1988), 405-421.

Voi91 M. Voit, On the dual space of a commutative hypergroup, Arch. Math. (Basel) 56 (1991), 380-385.

Voi06 M. Voit, Bessel convolutions on matrix cones: algebraic properties and random walks, Preprint (2006), math.CA/0603017.

Wat66 G. N. Watson, A treatise on the theory of Bessel functions (Cambridge University Press, Cambridge, 1966).

Margit Rösler roesler@math.tu-clausthal.de

Institut für Mathematik, TU Clausthal, Erzstr. 1, D-38678 Clausthal-Zellerfeld, Germany 